\title{
The deuterium abundance in Jupiter and Saturn from ISO-SWS observations
}

\author{
E. Lellouch ${ }^{1}$, B. Bézard ${ }^{1}$, T. Fouchet ${ }^{1}$, H. Feuchtgruber ${ }^{2}$, T. Encrenaz ${ }^{1}$, and T. de Graauw ${ }^{3}$ \\ 1 DESPA, Observatoire de Paris, 5 place Jules Janssen, 92195 Meudon Cedex, France \\ 2 Max-Planck-Institut für Extraterrestrische Physik, 85748 Garching, Germany \\ 3 Space Research Organization of the Netherlands and Kapteyn Institute PO Box 800, 9700 AV Groningen, \\ The Netherlands
}

Received 22 December 2000 / Accepted 14 February 2001

\begin{abstract}
Observations with the Short Wavelength Spectrometer (SWS) onboard the Infrared Space Observatory (ISO) are used to determine the D/H ratio in Jupiter's and Saturn's atmospheres. The D/H ratio is measured independently in hydrogen (i.e. from the $\mathrm{HD} / \mathrm{H}_{2}$ ratio) and methane (from $\mathrm{CH}_{3} \mathrm{D} / \mathrm{CH}_{4}$ ). Observations of the HD $R(2)$ and $R(3)$ rotational lines at 37.7 and $28.5 \mu \mathrm{m}$, of the $\mathrm{H}_{2} S(0)$ and $S(1)$ quadrupolar lines at 17.1 and $28.2 \mu \mathrm{m}$, of the methane $\nu_{4}$ band at $7.7 \mu \mathrm{m}$, and of the $\mathrm{CH}_{3} \mathrm{D} \nu_{6}$ band at $8.6 \mu \mathrm{m}$ are analyzed jointly, allowing a retrieval of thermal profiles and molecular abundances. On each planet, the $\mathrm{D} / \mathrm{H}$ determinations from $\mathrm{H}_{2}$ and $\mathrm{CH}_{4}$ give consistent results, but the accuracy is not sufficient to precisely determine the enrichment factor of $\mathrm{D} / \mathrm{H}$ in methane. Combining these determinations, we obtain the following values for the $\mathrm{D} / \mathrm{H}$ ratio in hydrogen: $(\mathrm{D} / \mathrm{H})_{\mathrm{H}_{2}}=(2.25 \pm 0.35) 10^{-5}$ in Jupiter and $\left(1.70_{-0.45}^{+0.75}\right) 10^{-5}$ on Saturn. These values are consistent with and somewhat more accurate than most earlier values. Comparing with inferences of protosolar D/H from solar wind measurements, it is confirmed that Jupiter is a reliable indicator of the protosolar $\mathrm{D} / \mathrm{H}$ ratio. The protosolar deuterium abundance inferred from the jovian value, $(2.1 \pm 0.4) 10^{-5}$, indicates a minor decrease of the $\mathrm{D} / \mathrm{H}$ ratio, over the last 4.55 Gyr, in the part of the Galaxy where the Solar System was formed. Although the error bars overlap, most measurements, including ours, may point to a slightly smaller D/H ratio in Saturn's atmosphere than in Jupiter's, a surprising result which needs confirmation.
\end{abstract}

Key words. planets and satellites: Jupiter - infrared: solar system

\section{Introduction}

An important constraint for models of galactic evolution is provided by the deuterium to hydrogen $(\mathrm{D} / \mathrm{H})$ ratio. Since deuterium in stars is converted to ${ }^{3} \mathrm{He}$, the $\mathrm{D} / \mathrm{H}$ ratio in the Universe is expected to decrease monotonically. In principle, a comparison of the $\mathrm{D} / \mathrm{H}$ ratio in the current nearby Local Interstellar Medium (LISM) with its value $4.55 \mathrm{Gyr}$ ago in the region of the interstellar medium where the Solar System was formed (the "protosolar value") provides a quantitative information on its variation with time in our galaxy, and possibly provides constraints on the primordial $\mathrm{D} / \mathrm{H}$.

While recent measurements of the $\mathrm{D} / \mathrm{H}$ ratio in the LISM seem to converge toward a single, relatively accurate value ((1.5 \pm 0.1$) 10^{-5}$; Linsky 1998; Sahu et al. 1999), the protosolar value remains more uncertain and requires measurements at appropriate places in the Solar System. One method consists of measuring the ${ }^{3} \mathrm{He} /{ }^{4} \mathrm{He}$ in the

Send offprint requests to: E. Lellouch, e-mail: emmanuel.lellouch@obspm.fr solar wind, to correct it for modifications occuring in the Sun's corona and chromosphere and for evolution in the solar interior, and to subtract the contribution of protosolar ${ }^{3} \mathrm{He}$ (e.g. Geiss 1993; Geiss \& Gloeckler 1998). The latter has usually been estimated from the ${ }^{3} \mathrm{He} /{ }^{4} \mathrm{He}$ ratio in meteorites; recently the first measurement of ${ }^{3} \mathrm{He} /{ }^{4} \mathrm{He}$ in Jupiter (Mahaffy et al. 1998) has provided an alternative method. The other method consists of identifying the protosolar D/H ratio to its value in Jupiter and Saturn, where it can be measured. This is valid if the entire deuterium in these planets came directly from the gaseous nebula with a negligible contribution from sources of fractionated (enriched) deuterium such as icy planetesimals. This assumption has traditionnally been made, owing to the large mass of their atmospheres compared to the mass of their cores (e.g. Stevenson 1982). (Note however that based on the most recent interior models, Guillot (1999) estimates that the $\mathrm{D} / \mathrm{H}$ ratio in Jupiter and Saturn could be respectively $\sim 5-10 \%$ and $25-40 \%$ higher than the protosolar value). In contrast, Uranus and Neptune have more than half of their total mass contained in their ice-rich 
interior, and therefore exhibit larger $\mathrm{D} / \mathrm{H}$ ratios than the protosolar value (see Feuchtgruber et al. 1999 for the most recent measurements).

The majority of $\mathrm{D} / \mathrm{H}$ determinations in Jupiter's and Saturn's atmospheres have been derived from the comparative abundance of methane $\left(\mathrm{CH}_{4}\right)$ and deuterated methane $\left(\mathrm{CH}_{3} \mathrm{D}\right)$, measured spectroscopically both in the near and thermal infrared (Knacke et al. 1982; Kunde et al. 1982; Drossart et al. 1982; Courtin et al. 1984; Owen et al. 1986; Noll \& Larson 1991; Bjoraker et al. 1986; Carlson et al. 1993). Besides the uncertainty on $\mathrm{CH}_{4}$ itself, particularly in Saturn, the interpretation of the $\mathrm{CH}_{3} \mathrm{D}$ measurements in terms of the bulk deuterium abundance (i.e. in $\mathrm{H}_{2}$ ) is complicated by the existence of an isotopic exchange reaction between $\mathrm{H}_{2}$ and $\mathrm{CH}_{4}\left(\mathrm{HD}+\mathrm{CH}_{4} \rightleftarrows\right.$ $\mathrm{CH}_{3} \mathrm{D}+\mathrm{H}_{2}$ ) whose thermodynamics, kinetics and coupling with atmospheric dynamics are uncertain (e.g. Beer \& Taylor 1973a,b, 1978; Fegley \& Prinn 1988; Lecluse et al. 1996; Smith et al. 1996; Smith 1998). In Jupiter, additional measurements have been obtained from the detection of HD at visible wavelengths (Trauger et al. 1973; Smith et al. 1989; note that a couple of unconfirmed detections have been presented for Saturn), and most recently from the in situ measurement by the GCMS instrument of the Galileo Probe (Mahaffy et al. 1998).

The operation of the Infrared Space Observatory (ISO, Kessler et al. 1996) in 1995-1998, carrying two spectrometers (SWS, de Graauw et al. 1996; and LWS, Clegg et al. 1996) covering altogether the $2.3-180 \mu \mathrm{m}$ range gave a new opportunity to measure spectroscopically the $\mathrm{D} / \mathrm{H}$ ratio in the Giant Planets. The newest aspect is the accessibility to the rotational lines of HD at $112 \mu \mathrm{m}(R(0))$, $56 \mu \mathrm{m}(R(1)), 37.7 \mu \mathrm{m}(R(2))$, and $28.5 \mu \mathrm{m}(R(3))$ (Ulivi et al. 1991). While these lines could not be detected by Voyager/IRIS because of unsufficient spectral resolution and sensitivity, Bézard et al. (1986) predicted that some of them would be detectable by ISO. On this basis, the $R(0)$ to $R(2)$ lines (and, in Saturn's case, the $R(3)$ line) were systematically searched for by ISO. In addition, for Jupiter and Saturn, the SWS range encompassed the region of the $\nu_{6}$ and $\nu_{2}$ bands of $\mathrm{CH}_{3} \mathrm{D}$ near 8.6 and $4.6 \mu \mathrm{m}$, respectively, and of the $\nu_{4}$ band of $\mathrm{CH}_{4}$ near $7.7 \mu \mathrm{m}$, permitting a new measurement of the $\mathrm{CH}_{3} \mathrm{D} / \mathrm{CH}_{4}$ ratio. A further advantage is that for each planet, the SWS measurements provided simultaneous information on the mean atmospheric thermal profile, notably from continuum and $\mathrm{H}_{2}$ quadrupolar lines measurements, which is necessary for an accurate abundance determination. The SWS observations of HD in Uranus and Neptune have been fully analyzed by Feuchtgruber et al. (1999). For Jupiter and Saturn, preliminary reports of the $\mathrm{HD}$ and $\mathrm{CH}_{3} \mathrm{D}$ measurements have been given (Encrenaz et al. 1996; Griffin et al. 1996; Lellouch et al. 1996, 1998; de Graauw et al. 1997). We present here the complete analysis of the SWS observations relevant to the $\mathrm{D} / \mathrm{H}$ ratio in Jupiter and Saturn, using the HD $R(2)$ and $R(3)$ rotational lines and the $\mathrm{CH}_{3} \mathrm{D}$ $8.6 \mu \mathrm{m}$ band. The $\mathrm{CH}_{3} \mathrm{D} \nu_{2}$ band at $4.6 \mu \mathrm{m}$, although detected, is not used, for several reasons. First, this band has been observed in the past from Earth at a higher spectral resolution than ISO (Bjoraker et al. 1986; Noll \& Larson 1991). Second, preliminary fits of this spectral region, as observed by ISO/SWS, have been presented by Encrenaz et al. 1996; de Graauw et al. 1997). They indicate $\mathrm{CH}_{3} \mathrm{D}$ mixing ratios consistent with the values we report below, but very uncertain, in part because of the difficulty to fit the continuum level at 4.55-4.7 $\mu \mathrm{m}$ (Roos-Serote 1997; Roos-Serote et al. 1999). Third, in Saturn's case, the $\mathrm{CH}_{4}$ mixing ratio is considerably uncertain and cannot be measured along with $\mathrm{CH}_{3} \mathrm{D}$ at $5 \mu \mathrm{m}$, further increasing the uncertainty in the $\mathrm{D} / \mathrm{H}$ ratio.

\section{Observations}

SWS observations of Jupiter and Saturn were carried out on several dates between January 1996 and December 1997. Essentially, three observing modes (AOT 02, AOT 06 and AOT 07) were used. AOT 06 is a grating mode covering a complete instrumental band (see de Graauw et al. 1996). The bands used here cover the 7.0-12.0 $\mu \mathrm{m}$, 12-16.5 $\mu \mathrm{m}, 16.5-19 \mu \mathrm{m}, 19-29.5 \mu \mathrm{m}$ and 29-45 $\mu \mathrm{m}$ ranges. Note that grating observations of Jupiter longward of $\sim 30 \mu \mathrm{m}$ are saturated and therefore not usable. Observations in this mode include the $S(0)$ and $S(1)$ quadrupolar lines of $\mathrm{H}_{2}$, at 28.2 and $17.0 \mu \mathrm{m}$ respectively. AOT 02 is also a grating mode, but limited to $\sim 10$ resolution elements around a wavelength of interest. It was used to search for the HD $R(2)$ line at $37.7 \mu \mathrm{m}$ in Saturn. In these two grating modes, the instrument aperture is $14 \times 20^{\prime \prime}$ or larger (see Table 1 ). The slit was centered on the planet subearth point and its long axis oriented towards celestial north. As the polar angles of Jupiter and Saturn over $1996-1997$ were in the range $340^{\circ}-360^{\circ}$ and $2^{\circ}-5^{\circ}$ respectively, the slit was roughly aligned with the central meridians. For targets comparable in size to the instrument aperture, as are Jupiter and Saturn, the spectral resolution for these two modes is 1000-2200, depending on wavelength. The AOT 07 mode, a Fabry-Perot mode with a spectral resolution of $\sim 30000$, was used to further observe the $\mathrm{H}_{2}$ quadrupolar lines, and to search for $\mathrm{HD}$ $R(2)$ on Jupiter and Saturn; an attempt was also made at the HD $R(3)$ line $(28.5 \mu \mathrm{m})$ on Saturn. The FP aperture is $10 \times 39^{\prime \prime}$ shortward of $26 \mu \mathrm{m}$ and $17 \times 40^{\prime \prime}$ longwards. Table 1 summarizes the data used in this paper. Note that a few other, preliminary AOT 07 observations have been acquired (particularly the HD $R(2)$ observation discussed in Encrenaz et al. 1996; Lellouch et al. 1996), but are superseded by the data presented here

All data were processed within the SWS interactive analysis system, based on standard pipeline OLP V7.0 products. The data reduction followed the recommendations of Salama et al. (1997). As shown in Figs. 1 and 2, the two quadrupolar lines of $\mathrm{H}_{2}$ were detected in emission in Saturn and Jupiter, both in the AOT 06 and AOT 07 modes, except the $S(0)$ line on Jupiter, which was detected only in Fabry-Perot mode. Note that the interpretation of this latter observation, taken early in the 
Table 1. Observations

\begin{tabular}{|c|c|c|c|c|c|}
\hline Target & Date (Orbit) & Mode & Aperture & Line/Range & Resolution \\
\hline Jupiter & 25-May-1997 (556) & S06 & variable $^{1}$ & $7-29 \mu \mathrm{m}$ & 1050 at $7 \mu \mathrm{m} ; 1350$ at $9 \mu \mathrm{m} ; 1600$ at $17 \mu \mathrm{m} ; 1000$ at $28 \mu \mathrm{m}$ \\
\hline Jupiter & 08-Mar.-1996 (112) & $\mathrm{S} 07$ & $10^{\prime \prime} \times 39^{\prime \prime}$ & $\mathrm{H}_{2} S(0) 28.22 \mu \mathrm{m}$ & 30000 \\
\hline Jupiter & 25-May-1997 (556) & S07 & $10^{\prime \prime} \times 39^{\prime \prime}$ & $\mathrm{H}_{2} S(1) 17.01 \mu \mathrm{m}$ & 33000 \\
\hline Jupiter & 25-May-1997 (556) & $\mathrm{S} 07$ & $17^{\prime \prime} \times 40^{\prime \prime}$ & HD $R(2) 37.70 \mu \mathrm{m}$ & 31000 \\
\hline Saturn & 06-Dec.-1996 (386) & S06 & variable $^{1}$ & $7-29 \mu \mathrm{m}$ & 1300 at $7 \mu \mathrm{m} ; 1700$ at $9 \mu \mathrm{m} ; 1900$ at $17 \mu \mathrm{m} ; 1200$ at $28 \mu \mathrm{m}$ \\
\hline Saturn & 14-Dec.-1997 (760) & $\mathrm{S} 07$ & $17^{\prime \prime} \times 40^{\prime \prime}$ & $\mathrm{H}_{2} S(0) 28.22 \mu \mathrm{m}$ & 30000 \\
\hline Saturn & 13-Dec.-1997 (758) & $\mathrm{S} 07$ & $10^{\prime \prime} \times 39^{\prime \prime}$ & $\mathrm{H}_{2} S(1) 17.01 \mu \mathrm{m}$ & 33000 \\
\hline Saturn & 14-Dec.-1997 (760) & S07 & $10^{\prime \prime} \times 39^{\prime \prime}$ & $\mathrm{H}_{2} S(1) 17.01 \mu \mathrm{m}$ & 33000 \\
\hline Saturn & 11-Dec.-1996 (391) & $\mathrm{S} 02$ & $20^{\prime \prime} \times 33^{\prime \prime}$ & $\mathrm{HD} R(2) 37.70 \mu \mathrm{m}$ & 1450 \\
\hline Saturn & 17-Dec.-1996 (763) & $\mathrm{S} 07$ & $17^{\prime \prime} \times 40^{\prime \prime}$ & $\mathrm{HD} R(3) 28.50 \mu \mathrm{m}$ & 30000 \\
\hline
\end{tabular}

mission (Rev. 112), may be more problematic, because post-mission analysis has revealed a large $\left(7^{\prime \prime}-9^{\prime \prime}\right)$ pointing error in cross-dispersion direction (i.e. toward the pole). Fluxes have been tentatively corrected for this error, but with some unavoidable uncertainty. Note also that for both Jupiter and Saturn, the Fabry-Perot observation of the $\mathrm{H}_{2} S(1)$ line exhibits a blue wing (at 17.033$17.034 \mu \mathrm{m})$ that cannot be reproduced by models. FabryPerot scans are always taken in the same direction, from red to blue. The blue wing is due to a memory effect of the Si:Sb detector for strong lines fluxes, which also causes the observed peak intensity to be too low.

Regarding HD, the $R(2)$ line was detected in Jupiter (AOT 07) (Fig. 3) and Saturn (AOT02 (Fig. 4), but not AOT07) in both cases in absorption; in Saturn, the $R(3)$ line was also marginally detected, in emission (AOT 07, Fig. 4). In addition, the $7-12 \mu \mathrm{m}$ spectra, already presented in Encrenaz et al. (1996) and de Graauw et al. (1997) clearly show the $\nu_{6}$ band of $\mathrm{CH}_{3} \mathrm{D}$ at $8.6 \mu \mathrm{m}$.

For both Jupiter and Saturn, the absolute accuracy of the flux scale for the AOT06 measurements is estimated to be $\sim 15 \%$ at $\lambda=12-16 \mu \mathrm{m}$. Although the absolute calibration for point sources is generally better than this (e.g. typically $11 \%$ at $12-16 \mu \mathrm{m}$, Salama et al. 1997), Saturn appears to be comparable with the instrumental aperture, inducing an additional uncertainty due to the unknown exact beam profile and possible losses of flux due to pointing inaccuracies. Jupiter, being always larger than the AOT06 apertures, is unaffected in this respect. On the other hand, it is a very strong target, which may be subject to partial saturation. These various effects and the change of aperture size at $12 \mu \mathrm{m}$ induce a discontinuity of the measured flux at $12 \mu \mathrm{m}$. The $7-12 \mu \mathrm{m}$ portion of Jupiter's and Saturn's spectra were rescaled to ensure continuity of the spectra at $12 \mu \mathrm{m}$. The $12-16 \mu \mathrm{m}$ spectral range here provides our "reference" flux calibration because it is known to be more reliable than the $7-12 \mu \mathrm{m}$ band (affected to by detector non-linearities) and because the aperture slit is longer (see Table 1), which reduces signal losses in Saturn's case. The flux corrections of the $7-12 \mu \mathrm{m}$ band were $5 \%$ (upwards) for Saturn, and as much as $-30 \%$ (downwards) for Jupiter, this much larger factor being
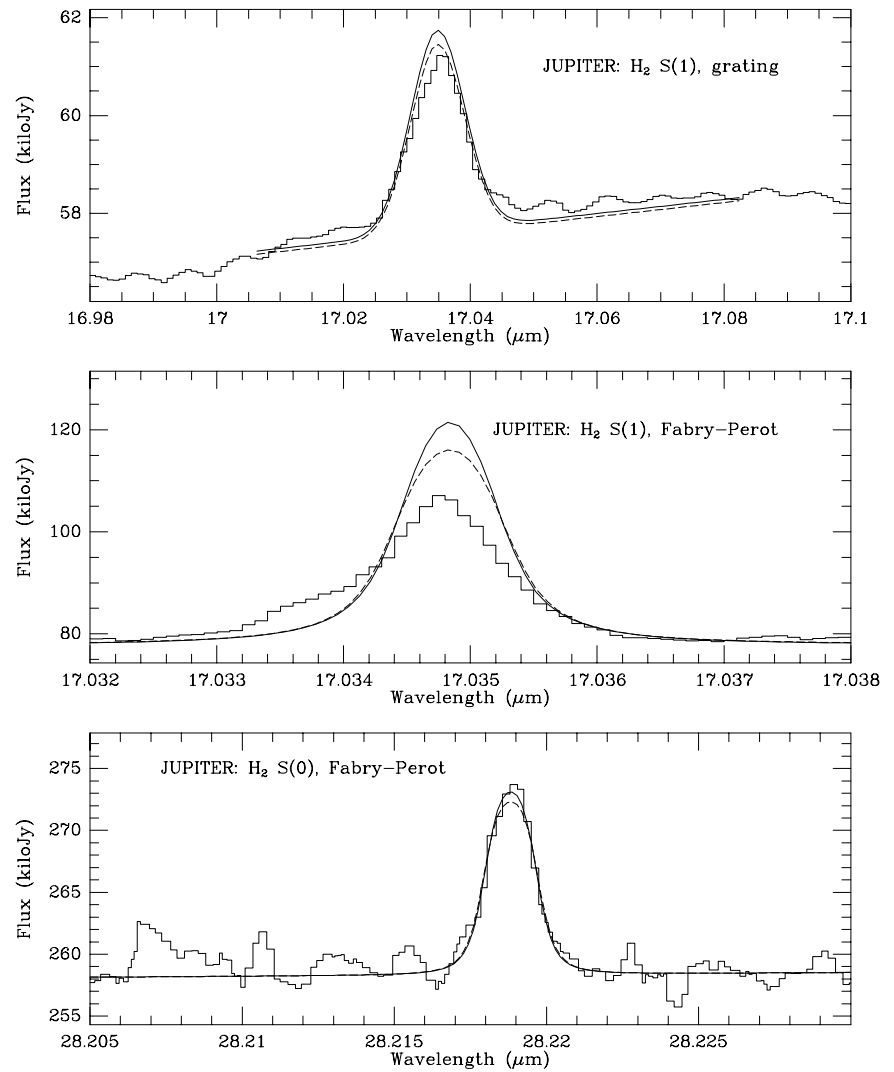

Fig. 1. The $\mathrm{H}_{2} S(0)$ and $S(1)$ line observations of Jupiter, in grating (AOT 06) and Fabry-Perot (AOT 07) modes. Observations are compared with models based either on the nominal thermal profile inferred from $\mathrm{CH}_{4}$ observations (solid line) or on the alternate profile best suited to the $\mathrm{H}_{2}$ lines (dashed line, see text). The flux scales refer to the model. In grating mode, the $S(1)$ flux has been rescaled by 1.085 . The Fabry-Perot mode data provide only line-to-continuum information

related to more important detector non-linearity effects at large fluxes.

At longest wavelengths $(\lambda>29.5 \mu \mathrm{m})$ the absolute calibration uncertainty increases to about $30 \%$, due to the lower number of usable calibration sources and to the more severe impact of uncertainties in the beam profile. For reasons explained in Moses et al. (2000a), the 

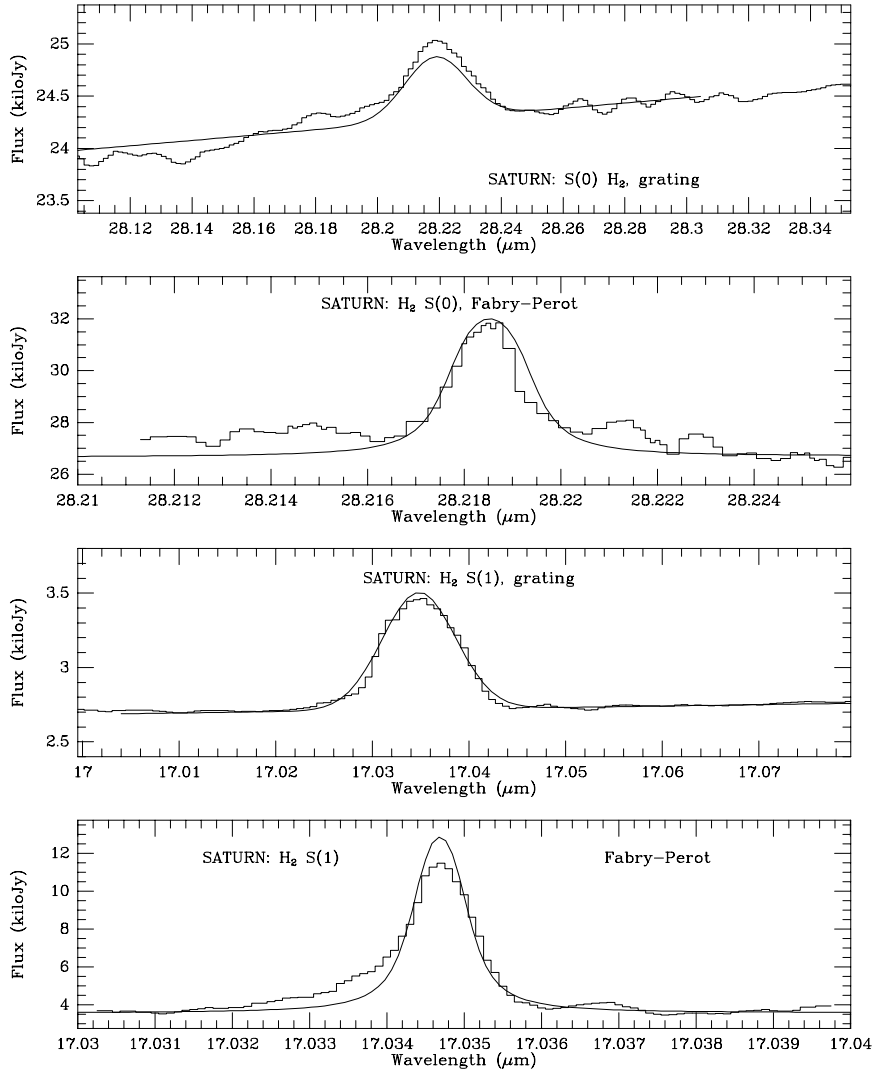

Fig. 2. The $\mathrm{H}_{2} S(0)$ and $S(1)$ line observations of Saturn, in grating (AOT 06) and in Fabry-Perot (AOT 07) modes. In each case, the observations are compared with models based on the nominal, cloud-free atmospheric model (see Fig. 6). The flux scale refers to the models. In grating mode, the $S(0)$ and $S(1)$ fluxes have been rescaled by 1.018 and 0.983 , respectively. The Fabry-Perot mode data provide only line-to-continuum information

absolute calibration of the AOT02 measurements is even more uncertain and the local slope of the continuum is not reliable. The same is true for the AOT07 measurements. In practice, only the line-to-continuum ratio can be used for observations in these latter two modes. Note finally that AOT07 observations shortward of $29 \mu \mathrm{m}$ suffer from "continuum leakage", i.e. the mixing of flux in the range of interest with flux originating from nearby continuum regions. This leakage, however, is well characterized: it leads to an apparent decrease (by $10 \%$ at $17 \mu \mathrm{m}$ and $20 \%$ at $28 \mu \mathrm{m}$ ) of the line-to-continuum ratios, which was accounted for in the modelling.

An additional complication for Saturn is the presence of thick rings that obscure a fraction of the planet. We calculated that the surface of the main rings in the SWS aperture amounted to only $3 \%$ of that from Saturn at the time of the observations. In addition, the brightness temperature of the rings is lower than Saturn's (Hanel et al. 1981). Thermal emission from the rings is then negligible and was not included in the modelling. On the other hand, reflected sunlight is weak but detectable at short wavelengths. We found that a grey geometric albedo of 0.15 for the rings, adding a flux of about $10 \mathrm{Jy}$ around

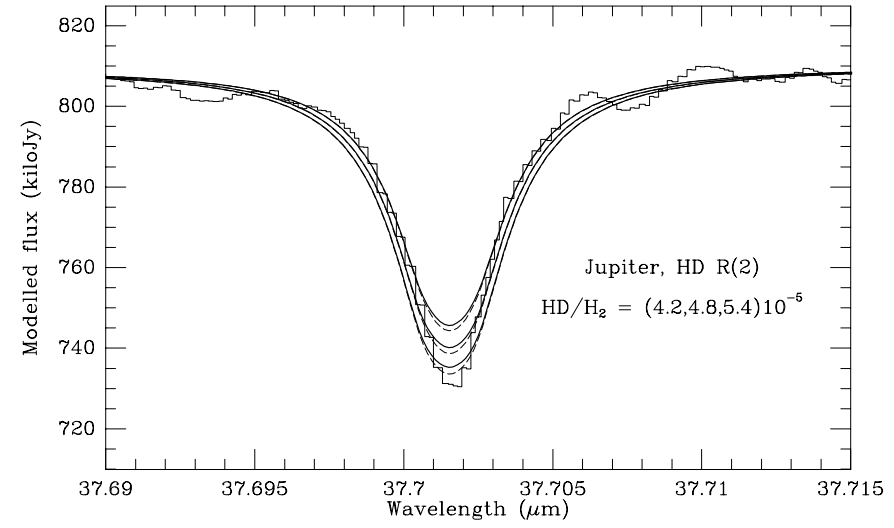

Fig. 3. The HD $R(2)$ line observation of Jupiter, compared with models with (from bottom to top) $\mathrm{HD} / \mathrm{H}_{2}=(4.2,4.8$, 5.4) $10^{-5}$. Solid lines: nominal thermal profile (solid line of Fig. 11). Dashed lines: alternate thermal profile (short-dashed line of Fig. 11). The flux scale refers to the models, as the data provide only line-to-continuum information

$7 \mu \mathrm{m}$, allowed us to reproduce the lowest flux levels observed shortward of $7.3 \mu \mathrm{m}$ where the thermal emission from Saturn is essentially zero. This ring contribution is negligible beyond $10 \mu \mathrm{m}$.

\section{Analysis}

The observations were analyzed by means of standard multilayer radiative non-scattering transfer models, including the collision-induced absorption of $\mathrm{H}_{2}-\mathrm{He}-\mathrm{CH}_{4}$ and all relevant molecular line opacities, namely $\mathrm{PH}_{3}$, $\mathrm{NH}_{3}, \mathrm{CH}_{4}, \mathrm{CH}_{3} \mathrm{D}, \mathrm{C}_{2} \mathrm{H}_{2}, \mathrm{C}_{2} \mathrm{H}_{6}, \mathrm{H}_{2}$ and HD. Line parameters were taken from the 1997 GEISA line databank (Jacquinet-Husson et al. 1999), except for $\mathrm{H}_{2}$ and HD. Line parameters for the quadrupolar lines of $\mathrm{H}_{2}$ were taken from Jennings et al. (1987) and Poll \& Wolniewicz (1978). For HD, spectroscopic parameters were calculated from Ulivi et al. (1991) and Drakoupolos \& Tabisz (1987a, 1987b). We estimate that the line intensities are accurate to within $10 \%$. In addition, because the $\mathrm{H}_{2}$ and $\mathrm{HD}$ rotational levels have relatively high energies (265 and $89 \mathrm{~cm}^{-1}$ respectively for the first levels), due to large rotational constants, the rotational partition function for these molecules $(Q)$ departs non-negligibly from the $T^{1}$ dependence usually valid for linear molecules. We found that at Jupiter's and Saturn's temperatures, $Q$ can be described by a $T^{0.95}$ and $T^{0.89}$ dependence for $\mathrm{H}_{2}$ and HD, respectively. The GEISA linelist for the $\nu_{4}$ band of $\mathrm{PH}_{3}$ was corrected for an error in the treatment of the transitions having $K^{\prime}=0$ and $K^{\prime \prime}=1$. Such transitions appear there as a doublet $\left(l^{\prime}= \pm 1\right)$, whereas only one component is allowed from selection rules. We then retained only one transition as given in Table II of Tarrago et al. (1981). $\mathrm{H}_{2^{-}}$ and He-broadened line halfwidths come from Levy et al. (1993) for $\mathrm{PH}_{3}$, Brown \& Peterson (1994) for $\mathrm{NH}_{3}$, and from a compilation by Linda Brown (private communication) for $\mathrm{CH}_{4}$. For $\mathrm{CH}_{3} \mathrm{D}$, we used the same halfwidths as $\mathrm{CH}_{4}$. To describe the linewings of $\mathrm{CH}_{4}$ and $\mathrm{CH}_{3} \mathrm{D}$, we used 

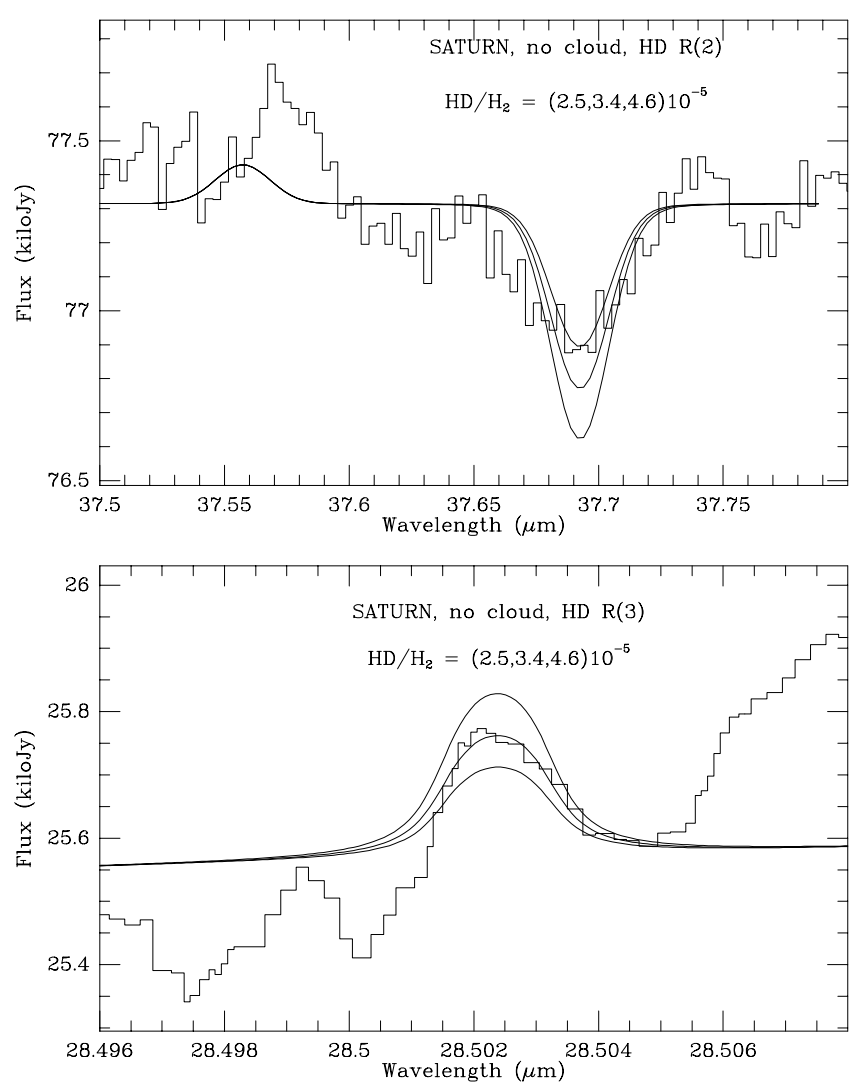

Fig. 4. The HD $R(2)$ and $R(3)$ line observations of Saturn (histograms), are compared with cloud-free models with (from bottom to top) $\mathrm{HD} / \mathrm{H}_{2}=(2.5,3.4,4.6) 10^{-5}$. The flux scales refer to the model, as the data provide only line-to-continuum information. Virtually identical line profiles can be obtained with the $\tau=1$ cloud case, by using $\mathrm{HD} / \mathrm{H}_{2}$ mixing ratios approximately $18 \%$ larger

a sublorentzian profile as determined by Hartmann et al. (2000) for the $\mathrm{CH}_{4} \nu_{3}$ band. For ammonia and phosphine, we simply used a Lorentz profile up to a cutoff distance of $20 \mathrm{~cm}^{-1}$.

We assumed an ortho-to-para ratio of $\mathrm{H}_{2}$ defined by local equilibrium in the calculation of both the $\mathrm{H}_{2}-\mathrm{He}$ continuum and the $\mathrm{H}_{2}$ quadrupolar lines. The helium mole fraction was taken to be 0.1342 in Jupiter (von Zahn et al. 1998) and 0.1183 in Saturn (Conrath \& Gautier 2000). In some cases, the atmospheric models also included absorbing layers meant to account for the presence of ammonia clouds, as detailed below.

Synthetic spectra were calculated monochromatically, integrated over viewing angles at the planets when necessary, and convolved with the instrumental profiles at the respective wavelengths. In the case of the grating observations, Saturn models were calculated from an integration over all airmasses in plane-parallel geometry (i.e. using the exponential integral $E_{2}$ ). This is only an approximation for the observations at $\lambda \leq 29 \mu \mathrm{m}$ for which the slit width is actually slightly smaller than Saturn's diameter, but the accuracy of the geometrical model is anyway limited by the uncertainty in the actual SWS beam profile and our neglect of Saturn's oblateness. For Jupiter, calculations for grating observations were performed for a single airmass, averaged over the slit (1.08 at $7-12 \mu \mathrm{m} ; 1.13$ for $\mathrm{H}_{2} S(1)$ ). The case of the Fabry-Perot observations is more delicate, because with equatorial velocities of typically $10 \mathrm{~km} \mathrm{~s}^{-1}$, rotational smearing contributes an additional broadening of $\sim 17 \mathrm{~km} \mathrm{~s}^{-1}$, equivalent to an effective resolving power of $\sim 18000$. The effect was included by partitioning the planetary disks into a square grid of regular spacing (40 elements along a diameter) and calculating a synthetic spectrum at each point within the instrumental aperture. In practice, this was done by spline interpolation in airmass on spectra precalculated at eight discrete airmasses at the planet $(1,1.1,1.3,1.8,2.5,4,8$ and 20$)$. These elementary synthetic spectra were then shifted in frequency to account for the local projected diurnal velocity and finally summed with appropriate weights. Again, Saturn's oblateness was not considered.

For both planets, the first step was to establish a thermal profile adequate for the $\mathrm{HD}$ and $\mathrm{CH}_{3} \mathrm{D}$ fitting. For this, we combined information from our AOT06 spectra with other constraints from various sources.

\subsection{Saturn}

\subsubsection{Thermal profile}

In Saturn's case, we followed the procedures outlined by Moses et al. (2000a, 2000b). In the 0.4-500 mbar range, the temperature profile is a modified version of the Voyager 2 ingress radio science (RSS) occultation profile (Lindal et al. 1985). Temperature adjustments were introduced in order to match the AOT06 spectrum itself shortward of $29 \mu \mathrm{m}$. The $\mathrm{H}_{2}-\mathrm{He}$ continuum at $13-29 \mu \mathrm{m}$ and in microwindows between 9 and $11 \mu \mathrm{m}$ constrains the temperature at tropospheric levels between 80 and 600 mbar. At levels deeper than 500 mbar, the temperature profile follows an adiabatic lapse rate corresponding to the "frozen equilibrium" case for the $\mathrm{H}_{2}$ ortho-to-para ratio. The $S(0)$ and $S(1)$ lines of $\mathrm{H}_{2}$ sound the middle stratosphere. Monochromatic contribution functions, defined as in Moses et al. (2000a), and calculated at line center and in the adjacent continua, are shown in Fig. 5. They illustrate that while the continuum is formed near 100 mbar for both lines, the $S(0)$ and $S(1)$ line centers probe respectively the 5 and 1.5 mbar region, with contribution functions extending (full width at half maximum) over three pressure scale heights. The Q-branch of the $\nu_{4}$ methane band at $7.66 \mu \mathrm{m}$ constrains to some extent the stratospheric temperature in the 0.5 -mbar region (see below Sect. 3.1.2). At pressures less than 0.4 mbar, the temperature profile was primarily based on stellar occultation measurements (Hubbard et al. 1997; see Moses et al. 2000b).

Saturn's spectrum longward of $30 \mu \mathrm{m}$ may be affected by cloud opacity (Conrath \& Pirraglia 1983; Courtin et al. 1984); however, the latter is uncertain and may be temporally and spatially variable. Therefore, we considered 

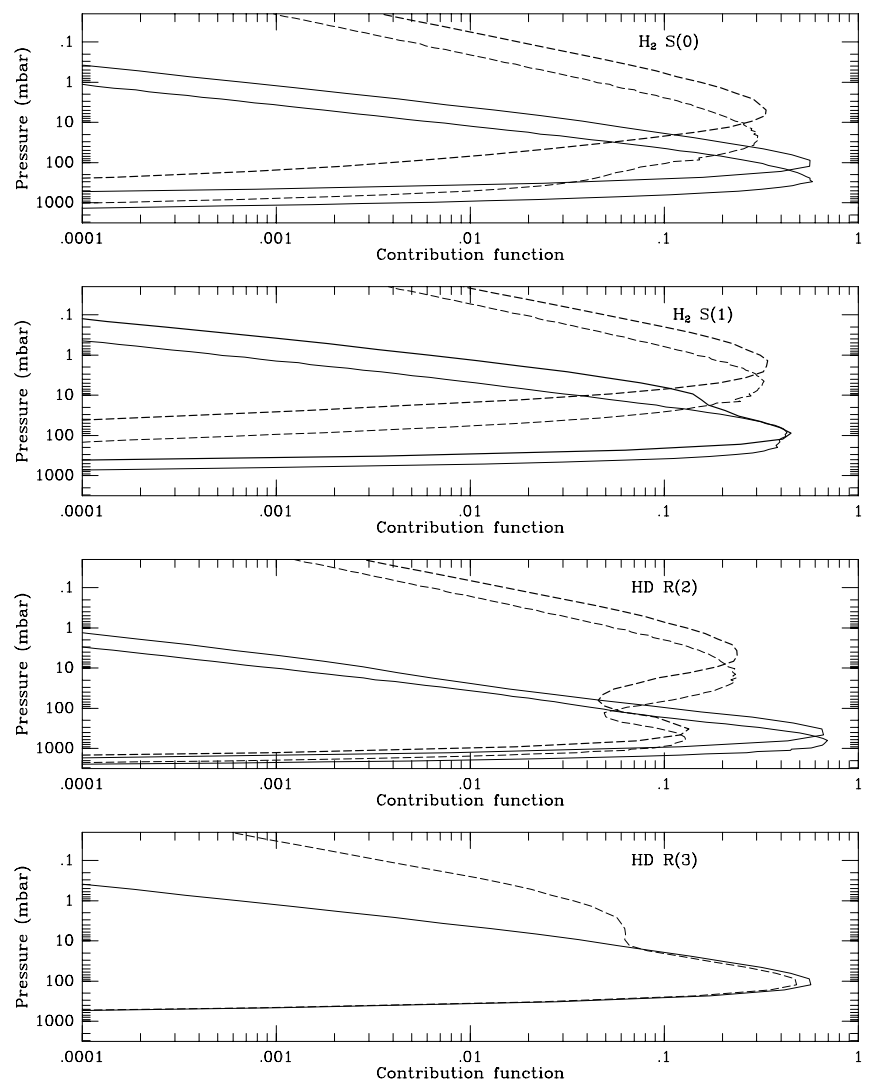

Fig. 5. Contribution functions at line center (dashed lines) and in the adjacent continuum (solid lines) of the $\mathrm{H}_{2} S(0), \mathrm{H}_{2} S(1)$, and HD $R(2)$ and $R(3)$ lines at Jupiter (thin lines) and Saturn (thick lines)

separately two cases: (i) a cloud-free atmospheric model and (ii) a model including a purely absorbing cloud, mimicking the $\mathrm{NH}_{3}$ cloud, which was introduced by specifying a cloud base at 1 bar, a cloud top at 300 mbar, a cloud scale height $H_{\mathrm{cl}}$ equal to the gas scale height, and a cloud optical depth equal to 1 . A cloud having these parameters was also used by Courtin et al. (1984) in their analysis of a large sample of Voyager /IRIS spectra. For each model, the thermal profile was retrieved as indicated above, making use of micro spectral windows that probe the 600-mbar region between 10 and $11 \mu \mathrm{m}$ (Fig. 6). Note that although, in principle, the continuum longward of $30 \mu \mathrm{m}$ could also be used to constrain the temperature and cloud optical depth around 500 mbar, the absolute calibration of the ISO data in this range is too uncertain for this purpose. The "cloudy" profile is warmer than the "cloud-free" profile by about $4 \mathrm{~K}$ at $730 \mathrm{mbar}$, a difference similar to the latitudinal contrast observed by Voyager/IRIS in the temperature field at this pressure level (Conrath \& Pirraglia 1983).

In both cases, the selected thermal profile allows an overall satisfactory match of the continuum level and of the $\mathrm{H}_{2}$ quadrupolar emissions (Fig. 2), both in the grating and Fabry-Perot observing modes. Yet, while the agreement is very good for the $S(1)$ line, especially in grating mode, the fits are not fully satisfactory for the $S(0)$

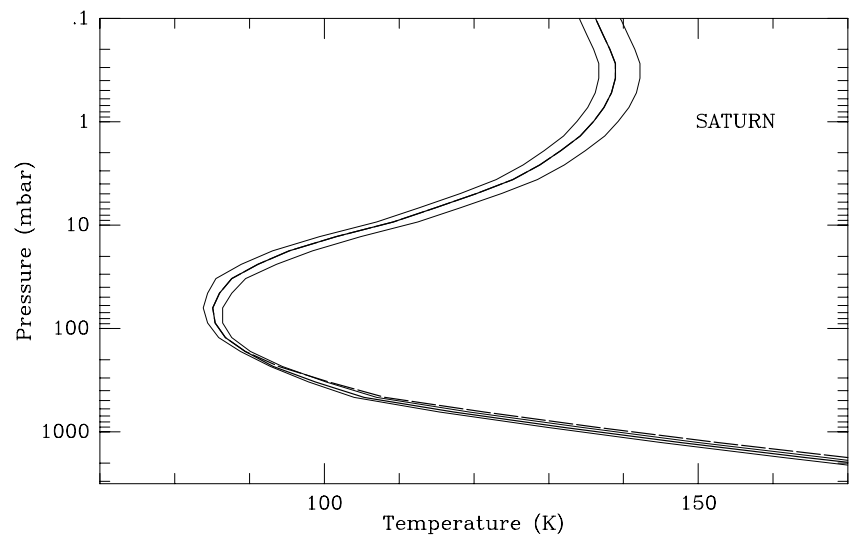

Fig. 6. Saturn's temperature profiles used in this study. Solid thick line: cloud-free profile. Long-dashed line: profile derived for a cloud opacity $\tau=1$. Solid thin lines: Warm and cold profiles, obtained after rescaling the observed spectra by $\pm 15 \%$ (see text)

line, which appears to be underestimated by the model in grating mode and overestimated in Fabry-Perot mode. The $S(0)$ data themselves appear to be mutually inconsistent and the model offers a compromise. We also note that our assumption of local equilibrium for the orthopara ratio may not be entirely valid; such departures have been observed in the tropospheres of all four Giant Planets (Conrath et al. 1998).

\subsection{2. $\mathrm{HD} / \mathrm{H}_{2}$ and $\mathrm{CH}_{3} \mathrm{D} / \mathrm{CH}_{4}$ abundance determinations}

The radiative transfer code was then applied to the HD, $\mathrm{CH}_{4}$ and $\mathrm{CH}_{3} \mathrm{D}$ observations. HD was assumed to be uniformly mixed with altitude. The distribution of $\mathrm{CH}_{4}$ and $\mathrm{CH}_{3} \mathrm{D}$ takes into account a depletion due to mass separation above the homopause level. All the $\mathrm{CH}_{4}$ and $\mathrm{CH}_{3} \mathrm{D}$ profiles tested below were deduced from those of Moses et al. (2000a) by applying a constant multiplicative factor.

HD. Monochromatic contribution functions in the center of the $R(2)$ lines of $\mathrm{HD}$ and in the adjacent continuum are shown in Fig. 5. The continuum is formed near 400 mbar. Although the HD line appears in absorption, its core probes a broad stratospheric region with maximum contribution at 4 mbar $(0.8-15$ mbar at FWHM). Similarly, the $R(3)$ line sounds the $0.5-10$ mbar range. These levels are similar to those sounded in the $\mathrm{H}_{2}$ quadrupolar lines, demonstrating the usefulness of the latter for modelling HD.

The best fit of the HD $R(2)$ and $R(3)$ lines is obtained for HD mixing ratios of $3.410^{-5}$ and $4.010^{-5}$ for the cloud free and $\tau=1$ cloud cases, respectively. Remarkably, the two HD lines give the same central value for the $\mathrm{HD} / \mathrm{H}_{2}$ mixing ratio, although the detection of the $R(3)$ is only marginal. The precision on these numbers due to $\mathrm{S} / \mathrm{N}$ limitations is ${ }_{-25}^{+35} \%$ (Fig. 4). In addition, there are 
uncertainties on the thermal profile associated to the absolute calibration uncertainties. The latter were estimated by determining extreme (i.e. warm and cold) temperature profiles. These were obtained by refitting the entire spectrum (i.e. the 9-11 $\mu \mathrm{m}$ continuum regions and the $\mathrm{H}_{2}$ lines) recalibrated by factors 1.15 and 0.85 , respectively. These thermal profiles, which pertain to the no cloud case, are shown in Fig. 6. Refitting the HD lines with the warm (resp. cold) thermal profile gave a variation of $-0.210^{-5}$ (resp. $+0.210^{-5}$ ) for the HD mixing ratio as determined from the $R(2)$ line, and a variation of $-0.410^{-5}$ (resp. $+0.310^{-5}$ ) for $R(3)$. Thus, the uncertainties due to calibration errors appear to be small (about 5 and $10 \%$ for the $R(2)$ and $R(3)$ line, respectively) compared to signal-tonoise limitations. Considering together the cloud-free and cloudy models, and accounting for the uncertainty in line intensities, the final $\mathrm{HD} / \mathrm{H}_{2}$ mixing ratio is $\left(3.7_{-1.2}^{+1.7}\right) 10^{-5}$. It is straightforwardly converted into the $\mathrm{D} / \mathrm{H}$ ratio in hydrogen through $(\mathrm{D} / \mathrm{H})_{\mathrm{H}_{2}}=\frac{1}{2} \frac{\mathrm{HD}}{\mathrm{H}_{2}}=1.85\left({ }_{-0.60}^{+0.85}\right) 10^{-5}$.

$\mathrm{CH}_{3} \mathrm{D} / \mathrm{CH}_{4}$. Measuring the $\mathrm{D} / \mathrm{H}$ ratio in the methane requires a determination of both the $\mathrm{CH}_{4}$ and $\mathrm{CH}_{3} \mathrm{D}$ abundances. The $\nu_{4}$ band of methane centered at $7.66 \mu \mathrm{m}$ exhibits many emission features originating from stratospheric levels. Emission in the Q-branch probes the 0.5mbar region. It is not too sensitive to the $\mathrm{CH}_{4}$ abundance and constrains the disk-averaged temperature around 0.5 mbar to be $\approx 139 \mathrm{~K}$. The P- and R-multiplets probe deeper, in the $0.5-3$ mbar region, and are more dependent on the $\mathrm{CH}_{4}$ profile. As temperature information is available in the same pressure range from the $S(0)$ and $S(1)$ $\mathrm{H}_{2}$ lines, it seems, in principle, possible to retrieve the $\mathrm{CH}_{4}$ mole fraction from the $\mathrm{P}$ and $\mathrm{R}$ emission features. However, the intensities of these features are affected by possible spatial variations of the temperature field because of the strong non-linearity of the Planck function with temperature at short wavelengths. For example, an equal average of emissions at 130 and $150 \mathrm{~K}$ produces a brightness temperature at $143.6 \mathrm{~K}$, almost $4 \mathrm{~K}$ higher than the average temperature. This is twice larger than the enhancement obtained by doubling the methane abundance from 3 to $610^{-3}$ ! This, in practice, precludes the direct use of the $\mathrm{P}$ and $\mathrm{R}$ branches to determine the $\mathrm{CH}_{4}$ mole fraction.

Horizontal variations of temperature are known to occur in Saturn's stratosphere and tropopause as a consequence of seasonal effects. This is attested by Voyager/IRIS measurements (Conrath \& Pirraglia 1983; Bjoraker et al. 1985) and ground-based spectral images in hydrocarbon bands (Gezari et al. 1989; Ollivier et al. 2000), and supported by seasonal models (Bézard \& Gautier 1985). To account for this non-uniformity, we used a simple two-region model in which we considered equal contributions from a "hot" and a "cold" hemisphere. The corresponding temperature profile in these regions depart from the nominal one (Fig. 6) by $\pm \Delta T$ above the 1-mbar level; deeper, the difference is reduced by half every

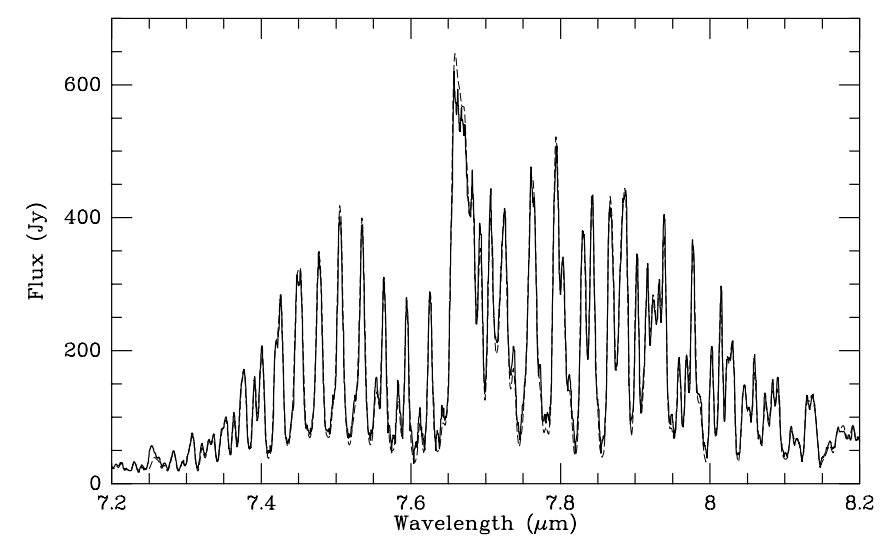

Fig. 7. The $\nu_{4}$ band of methane observed by ISO/SWS on Saturn (solid line) is compared with a synthetic spectrum (dashed line) using a $\mathrm{CH}_{4}$ mole fraction of $4.510^{-3}$. The synthetic spectrum is an average of spectra calculated with "hot" and "cold" temperatures profiles symmetrical about the nominal profile in Fig. 6 (see text)

pressure decade. For a given methane profile, $\Delta T$ was derived from the best fit of the $\mathrm{P}$ - and R-branches of the $\nu_{4}$ band. Figure 7 shows our best fit assuming a tropospheric $\mathrm{CH}_{4}$ mole fraction of $4.510^{-3}$ and using $\Delta T=10.8 \mathrm{~K}$. Such a temperature variation at $p \leq 1$ mbar may seem large but is not unreasonable in view of the $\pm \sim 5 \mathrm{~K}$ spatial contrasts observed near Saturn's tropopause (Conrath \& Pirraglia 1983). Equally good fits are obtained, for example, with mole fractions of $310^{-3}$ (with $\Delta T=12.4 \mathrm{~K}$ ) and $610^{-3}$ (with $\Delta T=9.4 \mathrm{~K}$ ). The way the $\mathrm{CH}_{4}$ abundance is determined is described below.

Beyond $8.1 \mu \mathrm{m}$, as $\mathrm{CH}_{4}$ opacity decreases, the atmosphere gets more transparent and emission from the troposphere becomes predominant. Phosphine is the main absorber between 8.1 and $11.3 \mu \mathrm{m}$ (e.g. de Graauw et al. 1997) and modelling of this region first requires to infer its vertical abundance profile. The present data provide information between 100 and 600 mbar approximately and we found that a $\mathrm{PH}_{3}$ profile with mole fractions of $610^{-6}$ up to the $600-$ mbar level, decreasing to $410^{-6}$ at $250 \mathrm{mbar}$ and $310^{-7}$ at 150 mbar, allowed us to reproduce well the numerous absorption features from the $\nu_{2}$ and $\nu_{4}$ bands. The fit however is not perfect, probably because of problems in the $\mathrm{PH}_{3}$ spectroscopic database. The $\mathrm{PH}_{3}$ profile we retrieve agrees well with those inferred by Weisstein \& Serabyn (1994) $\left(310^{-6}\right.$ up to 100 mbar), and Davis et al. (1996) $\left(710^{-6}\right.$ up to $\left.300 \mathrm{mbar}\right)$.

In the $\sim 8.15-8.5 \mu \mathrm{m}$ region, between the $\mathrm{PH}_{3}$ multiplets, the $\mathrm{CH}_{4}$ opacity is weak but not negligible and emission from the 600-1000 mbar levels is detected. The intensity of this emission then depends on the tropospheric methane abundance, in a vertical range where the temperature is independently constrained by the micro-windows between 10 and $11 \mu \mathrm{m}$. This provides a way of determining the $\mathrm{CH}_{4}$ tropospheric abundance similarly to Gautier et al.'s (1982) analysis for Jupiter. Figure 8 shows three calculations including mole fractions of $3,4.5$, and $610^{-3}$, 


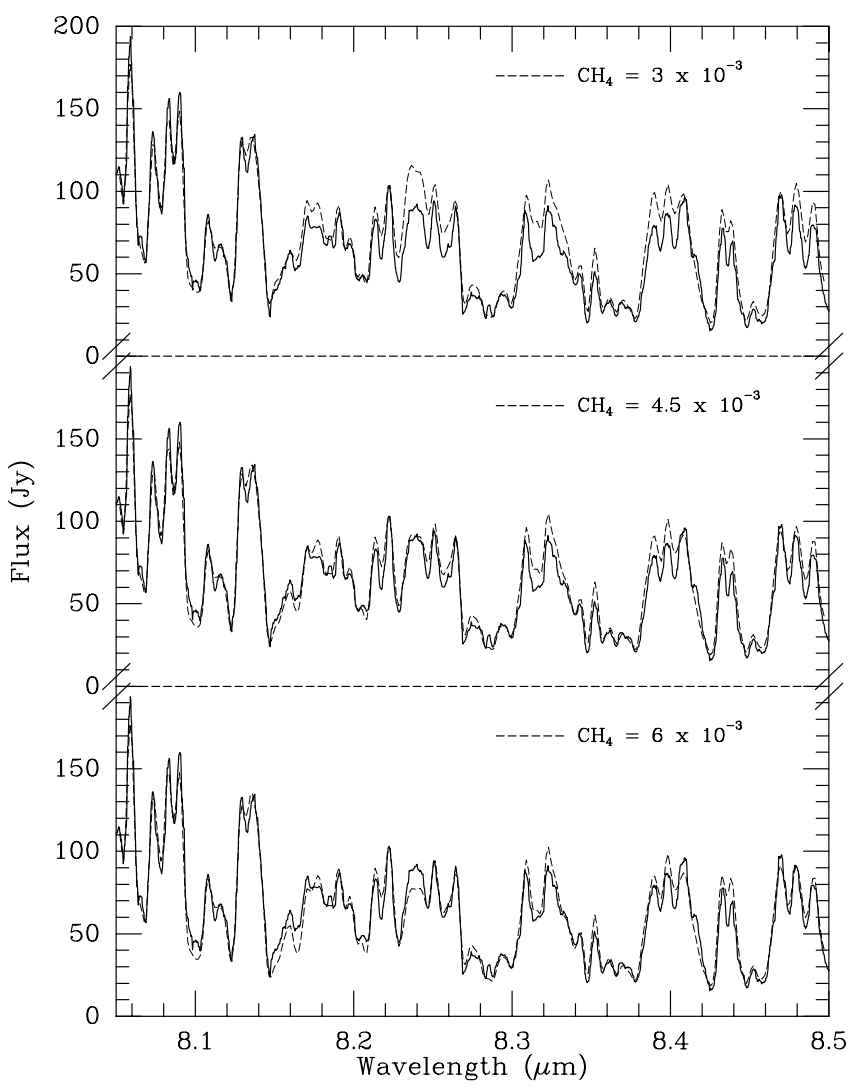

Fig. 8. The ISO/SWS Saturn spectrum in the wing of the Pbranch of the $\mathrm{CH}_{4}$ band (solid line) is compared to synthetic spectra calculated with three different $\mathrm{CH}_{4}$ abundance profiles and no cloud opacity (dashed lines). In each case, the temperature structure in the stratosphere is adjusted to reproduce the $\mathrm{CH}_{4}$ emission features. A $\mathrm{CH}_{3} \mathrm{D}$ mole fraction of $3.410^{-7}$ is assumed. The synthetic spectra mostly differ in relatively transparent regions where emission originates from the $\sim 600-1000$ mbar range

and no cloud opacity. In each case, the temperature structure was adjusted to correctly reproduce the emission features at shorter wavelengths, as discussed above. The intermediate $\mathrm{CH}_{4}$ abundance $\left(\begin{array}{ll}4.5 & 10^{-3}\end{array}\right)$ provides the best reproduction of the ISO spectrum and we consider that acceptable values range between 3.2 and $5.610^{-3}$. An atmospheric model with a $\tau=1$ cloud optical depth leads to a $\mathrm{CH}_{4}$ mole fraction $\sim 10^{-3}$ smaller, and values between 2.4 and $4.510^{-3}$ are then acceptable.

In summary, the existence of horizontal temperature variations considerably complicates the analysis. However, by combining information from the $\mathrm{H}_{2}$ quadrupolar lines, from the $\mathrm{CH}_{4} \nu_{4}$ extended emission at 7.4-8.1 $\mu \mathrm{m}$ and from the $\mathrm{CH}_{4}$ absorptions at $8.15-8.5 \mu \mathrm{m}$, we were able to obtain independently the disk-average stratospheric profile, a measurement of its spatial heterogeneity through the $\Delta T$ parameter, and the $\mathrm{CH}_{4}$ abundance in the wellmixed atmosphere.

The $\mathrm{CH}_{3} \mathrm{D}$ abundance was then derived from the 8.2-8.8 $\mu \mathrm{m}$ part of the spectrum. Figure 9 shows calculations in which the $\mathrm{CH}_{3} \mathrm{D}$ mixing ratio is successively

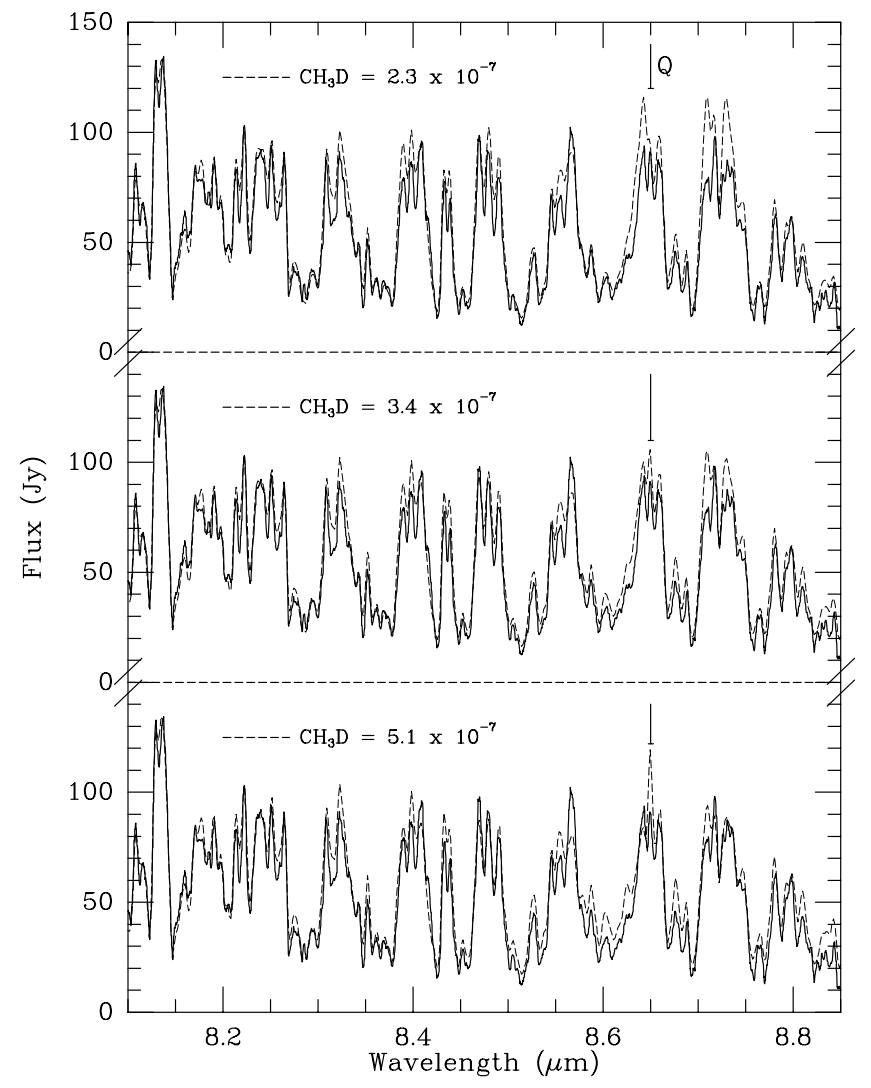

Fig. 9. The ISO/SWS Saturn spectrum in the $8.1-8.85 \mu \mathrm{m}$ region (solid line) is compared to synthetic spectra calculated with three different $\mathrm{CH}_{3} \mathrm{D}$ mole fractions, no cloud opacity, and a $\mathrm{CH}_{4}$ tropospheric mole fraction of $4.51^{-3}$ (dashed lines). The location of the Q-branch of the $\nu_{6}$ band of $\mathrm{CH}_{3} \mathrm{D}$ is indicated. Spectral features other than $\mathrm{CH}_{3} \mathrm{D}$ are due to $\mathrm{CH}_{4}$ and $\mathrm{PH}_{3}$

increased from top to bottom by $50 \%$ and $\mathrm{CH}_{4}$ kept to an abundance of $4.510^{-3}$. For large values of $\mathrm{CH}_{3} \mathrm{D}$ (lower panel), stratospheric emission from the Q-branch at $8.65 \mu \mathrm{m}$ becomes unacceptably prominent compared to the observations. In addition, stratospheric emission fills in the $\mathrm{PH}_{3}$ multiplets (e.g. around $8.60 \mu \mathrm{m}$ ), increasing the discrepancy with the ISO spectrum. A low abundance of $\mathrm{CH}_{3} \mathrm{D}$ (upper panel) increases the transparency of the atmosphere and yields a too large flux immediately shortward of the Q-branch as well as in the 8.70-8.73 $\mu \mathrm{m}$ interval. The intermediate mole fraction $\left(3.410^{-7}\right)$ yields the best compromise although significant discrepancies remain, possibly due to uncertainties in the $\mathrm{PH}_{3}$ spectroscopic database. We estimate that values between 2.5 and $4.510^{-7}$ are acceptable. Using the cloudy atmospheric model, the retrieved $\mathrm{CH}_{3} \mathrm{D}$ mole fraction shifts to $(3.0 \pm 1.0) 10^{-7}$.

Temperature profiles corresponding to a $\pm 15 \%$ calibration error (see above) lead to best fitting $\mathrm{CH}_{4}$ and $\mathrm{CH}_{3} \mathrm{D}$ abundances similar to the above values.

The $\mathrm{D} / \mathrm{H}$ ratio in the methane is given by $(\mathrm{D} / \mathrm{H})_{\mathrm{CH}_{4}}=$ $\frac{1}{4} \frac{\mathrm{CH}_{3} \mathrm{D}}{\mathrm{CH}_{4}}$. Combining all error sources, we derive that this ratio is equal to $\left(2.0_{-0.7}^{+1.4}\right) 10^{-5}$. Note that, since 
cloud models uncertainties affect the $\mathrm{CH}_{4}$ and $\mathrm{CH}_{3} \mathrm{D}$ abundances in a similar way, error bars on $(\mathrm{D} / \mathrm{H})_{\mathrm{CH}_{4}}$ are smaller than those obtained by independently combining individual uncertainties. This is the great advantage of measuring the two species in the same spectral range.

\subsection{Jupiter}

The analysis for Jupiter was similar to that for Saturn, but somewhat simpler in its principle. This stems from the fact that Jupiter's $\mathrm{CH}_{4}$ tropospheric abundance is well known, especially after the Galileo Probe measurements (Niemann et al. 1998) which confirmed the Voyager 1 spectroscopic determination (Gautier et al. 1982).

\subsubsection{Thermal profile}

A thermal profile suitable for the analysis of the HD and $\mathrm{CH}_{3} \mathrm{D}$ features was constructed by starting with and modifying the Galileo Probe entry profile (Seiff et al. 1998). Similar to Saturn, the $\mathrm{H}_{2}-\mathrm{He}$ continuum due to $\mathrm{H}_{2}-\mathrm{He}$ at $13-16 \mu \mathrm{m}$ and in microwindows between $\mathrm{CH}_{4}$ absorptions at 7-9 $\mu \mathrm{m}$ constrain the temperature between the tropopause and the $500 \mathrm{mbar}$ level. We found, however, that fitting the $7-9 \mu \mathrm{m}$ spectral region always required the presence of an attenuating cloud. Such a cloud was specified with a base at $900 \mathrm{mbar}$, and a scale height equal to 0.15 times the gas scale height (as in Conrath \& Gierasch 1986). The abundance and vertical profile of $\mathrm{NH}_{3}$, which impacts to some extent on the 7-9 $\mu \mathrm{m}$ spectrum, was inferred from the 9.5-11.5 $\mu \mathrm{m}$ region (see Fouchet et al. 2000a). The best fit was obtained for a thermal profile colder than the Galileo Probe profile, by $2.5 \mathrm{~K}$ at the tropopause and $0.5 \mathrm{~K}$ at $300 \mathrm{mbar}$, and a total cloud opacity $\tau=1.0$. Forcing the fit in the $7-9 \mu \mathrm{m}$ range without cloud attenuation not only implied unrealistic temperatures (colder by $10 \mathrm{~K}$ than the Galileo profile at $1 \mathrm{bar}$ ), but also irreversibly degraded the fit at the longest wavelengths, especially in the $9.5-11.5 \mu \mathrm{m}$ region. At levels deeper than 0.5 bar, the Galileo profile was adopted without modification for our nominal profile. In order to take into account possible uncertainties on the temperature at 1 bar, we also used two profiles respectively warmer and colder by $2 \mathrm{~K}$ at this pressure level. This required to modify the cloud opacity to values of 1.27 and 0.75 , respectively.

The stratospheric $\nu_{4}$ emission constrains the thermal structure from $\sim 1$ mbar to 35 mbar. We used a deep $\mathrm{CH}_{4}$ mole fraction of $1.810^{-3}$ (i.e. $\mathrm{CH}_{4} / \mathrm{H}_{2}=2.110^{-3}$; Niemann et al. 1998), and the vertical profile derived by Drossart et al. (1999) from the $\mathrm{CH}_{4}$ fluorescence at $3.3 \mu \mathrm{m}$. Similar to Saturn, the $S(0)$ and $S(1)$ lines of $\mathrm{H}_{2}$ sound the middle stratosphere (peak of the contribution functions at 25 and 5 mbar respectively; cf. Fig. 5). The vertical profile inferred from the $\mathrm{CH}_{4} \nu_{4}$ emission is shown as the solid line of Fig. 10. The corresponding fit is shown in Fig. 11. This fit is admittedly not entirely satisfactory. In particular,

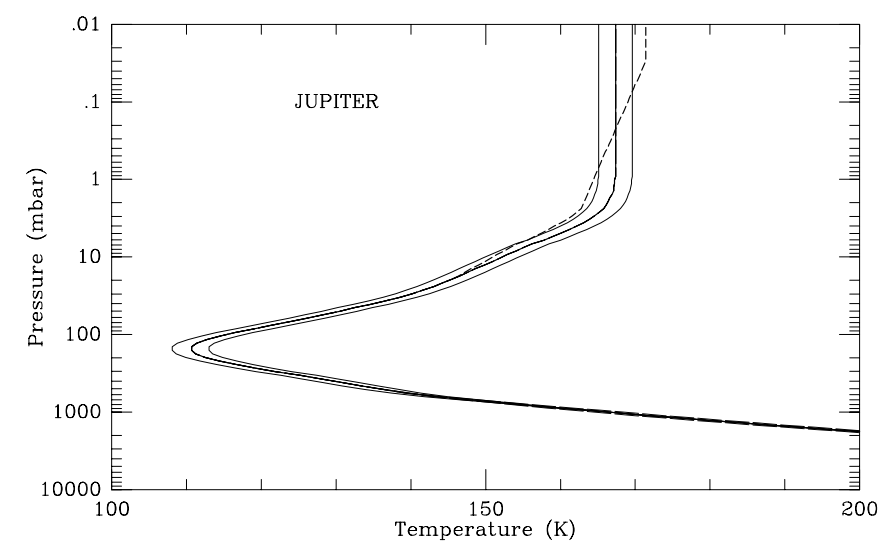

Fig. 10. Jupiter's thermal profiles used in this study. Solid thick line: nominal profile. Solid thin lines: warm and cold profiles, obtained after rescaling the observed spectra by $\pm 15 \%$. Long-dashed lines: profiles differing from the nominal profile by $\pm 2 \mathrm{~K}$ at 1 bar (see text). Short-dashed line: profile specifically suited to the $\mathrm{H}_{2}$ quadrupolar lines (see text)

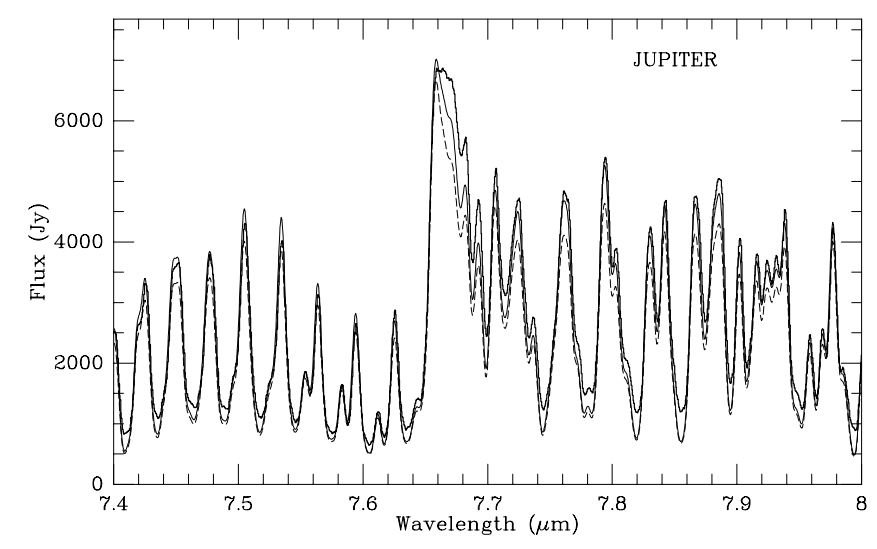

Fig. 11. The $\nu_{4}$ band of methane observed by ISO/SWS on Jupiter (thick solid line) compared with two synthetic spectra using a $\mathrm{CH}_{4}$ tropospheric mole fraction of $1.810^{-3}$. The thin solid line corresponds to the nominal thermal profile (solid line of Fig. 10) and the thin dashed line corresponds to the alternate profile (short-dashed line in Fig. 10)

the modelled Q branch appears too narrow, and the flux in the regions of minimum absorption between the individual $\mathrm{P}$ and $\mathrm{R}$ lines is underpredicted. Similar problems, already encountered in Fouchet et al. (2000b), are also apparent, although to a much lower degree, in the modelling of Saturn's spectrum (Fig. 7). They may result from (i) line mixing in the $\mathrm{Q}$ branch (ii) a solar-reflected contribution due to the presence of stratospheric hazes (iii) an instrumental shift of the zero level in Jupiter's spectrum.

This preferred model tends to overestimate the $\mathrm{H}_{2}$ quadrupolar emission. As a matter of fact, the observationally more uncertain $S(0)$ Fabry-Perot observation is well reproduced (Fig. 1). However, the $S(1)$ emission is somewhat overpredicted, both in grating and Fabry-Perot modes. As for Saturn, and as already noted by Fouchet et al. (2000b), departures from local equilibrium of the ortho-para ratio in Jupiter's stratosphere are possible; 
should that be the case, the best temperature model in the framework of our equilibrium approach should provide a compromise between the $S(0)$ and $S(1)$ lines. Such a model is shown in Fig. 1. The corresponding alternate temperature profile (short-dashed line in Fig. 10) is $3 \mathrm{~K}$ colder than the one inferred from the $\mathrm{CH}_{4} \nu_{4}$ emission alone. This, however, leads to an unacceptable degradation of the fit in the $\mathrm{CH}_{4} \nu_{4}$ band, not only in the $\mathrm{Q}$ branch at $7.66-7.70 \mu \mathrm{m}$, but also in most $\mathrm{P}$ and $\mathrm{R}$ lines (flux loss of $15-20 \%$ ).

We were not able to solve this dilemma. In particular, we noted that an increase of $20 \%$ in the methane abundance, as warranted by the current uncertainty of the $\mathrm{CH}_{4} / \mathrm{H}_{2}$ ratio (Niemann et al. 1998), has a negligible effect. As on Saturn, spatial variations of the thermal profile result in a selective increase of the $\mathrm{CH}_{4}$ emission. However, such variations are expected to be much smaller on Jupiter where seasonal effects are much less important. In addition, the SWS-grating field-of-view does not encompass Jupiter's high latitudes. Specifically, we found that fully reconciling the $\mathrm{H}_{2}$ emission with the $\mathrm{CH}_{4}$ emission would require dividing Jupiter's stratosphere into "hot" and "cold" regions of equal surface and departing by $\Delta T= \pm 12 \mathrm{~K}$ at 0.5 mbar from the mean temperature. This seems an unreasonable case, at it would, in particular, lead to a factor-of- 3 difference in the flux emitted by the hot and the cold regions. Ground-based observations at $7.54 \mu \mathrm{m}$ (Orton et al. 1992, G. S. Orton, priv. comm., 2000) reveal that mid-latitude spatial variability in the 0.1-10 mbar range remains well within the $\pm 5 \mathrm{~K}$ range. Thus, this difficulty to fit the entire Jupiter spectrum with a single atmospheric model remains to be elucidated.

We finally adopted the thermal profile inferred from the $\mathrm{CH}_{4} \nu_{4}$ emission as the nominal profile for the HD and $\mathrm{CH}_{3} \mathrm{D}$ studies. Nonetheless, in the case of $\mathrm{HD}$, we also used the alternate profile presented above. The nominal profile is also subject to uncertainties associated with the $\pm 15 \%$ absolute calibration uncertainty. The corresponding warm and cold profiles are also shown in Fig. 10. The associated cloud opacities are 0.73 and 1.20 , respectively.

\subsection{2. $\mathrm{HD} / \mathrm{H}_{2}$ and $\mathrm{CH}_{3} \mathrm{D} / \mathrm{CH}_{4}$ abundance determinations}

HD. Similarly to Saturn, contribution functions calculated in the center of the $R(2)$ line of HD and in the adjacent continuum indicate that the continuum formation occurs near 630 mbar (Fig. 5), while the HD core probes a broad region centered at 14 mbar and extending from 2.5 to 60 mbar at FWHM.

With the nominal thermal profile as discussed above, the best fit of the $R(2)$ line is achieved for a $\mathrm{HD} / \mathrm{H}_{2}$ mixing ratio of $4.810^{-5}$ (Fig. 4). The uncertainty on this number due to $\mathrm{S} / \mathrm{N}$ limitations is $\pm 0.610^{-5}$. This uncertainty must be convolved with the uncertainties linked to thermal profiles. The alternate profile described above induces a small change (less than $-0.210^{-5}$ ) in the
HD mixing ratio (Fig. 3). The warm and cold profiles resulting from the $\pm 15 \%$ calibration uncertainties result in a similar $\pm 0.210^{-5}$ change in HD. Finally, the $\pm 2 \mathrm{~K}$ uncertainty at 1 bar has an even smaller impact on $\mathrm{HD} / \mathrm{H}_{2}$ $\left(\sim 0.1510^{-5}\right)$. Quadratically adding the uncertainties, the jovian $\mathrm{HD} / \mathrm{H}_{2}$ mixing ratio is $(4.8 \pm 0.8) 10^{-5}$, providing $(\mathrm{D} / \mathrm{H})_{\mathrm{H}_{2}}=(2.4 \pm 0.4) 10^{-5}$.

$\mathrm{CH}_{3} \mathrm{D} / \mathrm{CH}_{4}$. Figure 12 shows the $8-9 \mu \mathrm{m}$ portion of the ISO spectrum, compared with three models based on the nominal thermal profile of Fig. 10 and having $\mathrm{CH}_{3} \mathrm{D}$ mole fractions of $0.8510^{-7}, 1.610^{-7}$ and $2.410^{-7}$, respectively. At our spectral resolution, $\mathrm{CH}_{3} \mathrm{D}$ tropospheric absorption impacts the spectrum both as discrete features, notably at $8.63,8.65$, and $8.70-8.73 \mu \mathrm{m}$, and as a continuum effect over the $8.2-8.8 \mu \mathrm{m}$ range. For this reason, for each value of the $\mathrm{CH}_{3} \mathrm{D}$ mole fraction, the cloud opacity was fine tuned to obtain an optimum match of the apparent pseudo-continuum at $8.56 \mu \mathrm{m}$. This cloud opacity varied from 0.9 for $\mathrm{CH}_{3} \mathrm{D}=2.410^{-7}$ to 1.1 for $\mathrm{CH}_{3} \mathrm{D}=$ $0.8510^{-7}$. The best fit is obtained for a $\mathrm{CH}_{3} \mathrm{D}$ mole fraction of $1.610^{-7}$, and we regard the (1.2-2.0) $10^{-7}$ range as giving acceptable fits. Error bars due to the $\pm 15 \%$ calibration uncertainty are small $\left( \pm 0.11^{-7}\right)$. The $\pm 2 \mathrm{~K}$ uncertainty at 1 bar induces a larger, but still weak, additional uncertainty of $\pm 0.210^{-7}$. This is linked to the required changes in the cloud transmission and ammonia profiles associated with the change in thermal profile (see Fouchet et al. 2000), in turn impacting on the $\mathrm{CH}_{3} \mathrm{D}$ abundance. Convolving these error bars, we obtain a $\mathrm{CH}_{3} \mathrm{D}$ mole fraction of $(1.6 \pm 0.5) 10^{-7}$. For a nominal $\mathrm{CH}_{4}$ mole fraction of $1.810^{-3}$, this gives a jovian $\mathrm{D} / \mathrm{H}$ ratio in methane, $(\mathrm{D} / \mathrm{H})_{\mathrm{CH}_{4}}=\frac{1}{4} \frac{\mathrm{CH}_{3} \mathrm{D}}{\mathrm{CH}_{4}}=(2.2 \pm 0.7) 10^{-5}$. We finally checked that the uncertainty in the $\mathrm{CH}_{4}$ abundance has no additional impact, because the temperature modification associated with a change in $\mathrm{CH}_{4}$ induces a similar change in $\mathrm{CH}_{3} \mathrm{D}$, leaving the $\frac{\mathrm{CH}_{3} \mathrm{D}}{\mathrm{CH}_{4}}$ essentially unchanged.

\section{Discussion}

In principle, the simultaneous determination of the $\mathrm{D} / \mathrm{H}$ ratio in methane and hydrogen provides a determination of the isotopic enrichment factor $f$ defined by:

$f=\frac{(\mathrm{D} / \mathrm{H})_{\mathrm{CH}_{4}}}{(\mathrm{D} / \mathrm{H})_{\mathrm{H}_{2}}}$.

However, our results, gathered in Table 2, are not accurate enough for this, since they lead to $f=0.9_{-0.4}^{+0.55}$ at Jupiter and $f=1.1_{-0.6}^{+1.7}$ at Saturn. The error bars are so large that these constraints are essentially useless, except for the upper limit $f<1.45$ at Jupiter. Thus, the observational determination of $f$, envisaged e.g. by Smith et al. (1996), is far beyond the capabilities of our ISO measurements, and would require a precision on the $\mathrm{CH}_{3} \mathrm{D}$ and HD abundances of order $5 \%$. We now discuss the available theoretical estimates of $f$. 


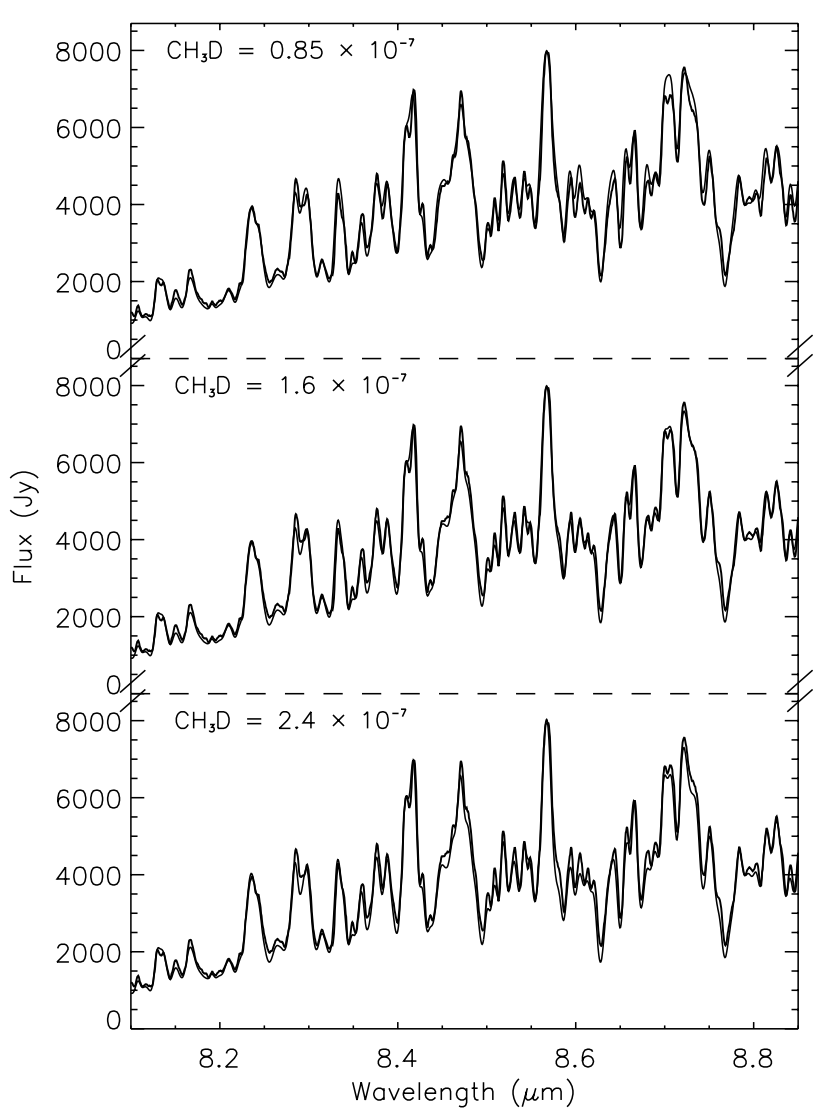

Fig. 12. The $8.1-8.85 \mu \mathrm{m}$ part of Jupiter ISO/SWS spectrum (thick solid line), compared with synthetic spectra (thin solid lines) calculated with three different $\mathrm{CH}_{3} \mathrm{D}$ mole fractions: (0.85, 1.6 and 2.4) $10^{-7}$. The $\mathrm{CH}_{4}$ tropospheric mole fraction is $1.810^{-3}$. The cloud opacities are $1.1,1.0$, and 0.9 , respectively

The value of the enrichment factor $f$ in Jupiter and Saturn depends not only on the chemical equilibrium of the isotopic exchange reaction between $\mathrm{CH}_{4}$ and $\mathrm{H}_{2}$, but also on the time scale for chemical equilibration $t_{f}$, and on the effect of atmospheric dynamics. In initial work (Beer \& Taylor 1973a; Beer \& Taylor 1978; Fegley \& Prinn 1988), the effect of dynamics was simply considered in the framework of a "quench level" theory. This is the level at which the enrichment factor equilibration time scale becomes longer than the diffusive mixing time scale, effectively "freezing" the equilibration reaction. Using this approach and an estimate of $t_{f}$ based on isotopic exchange measurements involving radicals, Fegley \& Prinn (1988) obtained $f=1.205$ for Jupiter and $f=1.229$ for Saturn. More recently, Lécluse et al. (1996) used actual laboratory measurements of the $\mathrm{CH}_{4}-\mathrm{H}_{2}$ isotopic exchange to estimate $t_{f}$. They computed the enrichment factor as a function of depth by assuming a vertical velocity profile $w(z)$, based on mixing length arguments (a "bulk motion" model), and obtained $f=1.25 \pm 0.12$ for Jupiter and $f=1.38 \pm 0.15$ for Saturn. Smith et al. (1996) (with an update by Smith 1998) adopted the $t_{f}$ value from Fegley \& Prinn (1988) and focussed on the effect of atmospheric dynamics. They critically examined the approach of Lécluse et al. as well as the alternate approach of a "diffusive mixing model", and instead proposed an "explicit diffusion model", in which atmospheric mixing is treated as the combined effect of many small-scale mixing events occuring at a random depth, characterized by a mean value $\bar{l}$. In this framework, they calculated the enrichment factor and examined its sensitivity to the value of $\bar{l}$ and to the dynamical regime (i.e. to the $w(z)$ profile, which can be estimated from mixing length theory, thermal wind equation, or plume dynamics). Their different models lead to $f=1.13-1.27$ for Jupiter and $f=1.15-1.33$ for Saturn.

On this basis, we here conservatively adopt $f=1.25 \pm$ 0.12 for Jupiter and $f=1.34 \pm 0.19$ for Saturn, which encompass the ranges of all previous studies. Even so, the uncertainty on $f(\sim 10 \%$ at Jupiter and $\sim 14 \%$ at Saturn $)$ is considerably smaller than that on our $(\mathrm{D} / \mathrm{H})_{\mathrm{CH}_{4}}$ ratios. Therefore, the latter can be combined with $f$ to provide another determination of $(\mathrm{D} / \mathrm{H})_{\mathrm{H}_{2}}$ (see Table 2). Averaging this determination with the direct measurement from $\mathrm{HD}$, we obtain our final $(\mathrm{D} / \mathrm{H})_{\mathrm{H}_{2}}$ ratios from ISO/SWS:

$$
\begin{aligned}
& (\mathrm{D} / \mathrm{H})_{\mathrm{H}_{2}}=(2.25 \pm 0.35) 10^{-5} \text { on Jupiter } \\
& (\mathrm{D} / \mathrm{H})_{\mathrm{H}_{2}}=\left(1.70_{-0.45}^{+0.75}\right) 10^{-5} \text { on Saturn. }
\end{aligned}
$$

As it is clear from Table 2, our values agree with most recent (post-1985) determinations. Regarding Jupiter, an overall consistency is reached between the $5 \mu \mathrm{m} \mathrm{CH}_{3} \mathrm{D}$ measurements of Bjoraker et al. (1986) $)^{1}$, the HD visible observations of Smith et al. (1989), the in situ Galileo measurement (Mahaffy et al. 1998) and our results. Only the value obtained by Carlson et al. (1993) from a reanalysis of the Voyager $5 \mu \mathrm{m}$ hot spot spectra is an outlier. At Saturn, besides a relatively inaccurate (50\% uncertainty) measurement at $1.6 \mu \mathrm{m}$ (Owen et al. 1986), the only recent $\mathrm{CH}_{3} \mathrm{D}$ measurement is that of Noll \& Larson (1991), who obtained a $\mathrm{CH}_{3} \mathrm{D}$ mole fraction $\left((3.3 \pm 1.5) 10^{-7}\right)$ entirely consistent with ours. Regarding HD, besides an upper limit obtained by Smith et al. (1989), the only published value is from an analysis of preliminary ISO/LWS observations (acquired during the ISO performance verification phase) of the HD $R(1)$ line at $56 \mu \mathrm{m}$. This gives $(\mathrm{D} / \mathrm{H})_{\mathrm{H}_{2}}=2.3_{-0.8}^{+1.2} 10^{-5}$, but this value must be viewed as provisional as better data are currently being processed and analyzed (G. Davis, priv. comm).

As mentioned in the introduction, Jupiter's deuterium content may represent a good estimate of the protosolar value. According to Guillot (1999), Jupiter's D/H ratio may still be enriched by some $5-10 \%$ above the protosolar nebula by the mixing of nebular gas with deuteriumrich ices during the planet's formation. Correcting the ISO value for this effect, we would propose $(D / H)_{\mathrm{ps}}=$ $(2.1 \pm 0.4) 10^{-5}$ as our best estimate of the protosolar

1 Bjoraker et al. (1986) actually published a low $\mathrm{D} / \mathrm{H}((1.2 \pm$ 0.5) $10^{-5}$ ) value at Jupiter, but this was largely based on too high adopted values for $\mathrm{CH}_{4}$ and $f$. 
Table 2. Recent (>1985) measurements of the D/H ratio in Jupiter and Saturn

\begin{tabular}{|c|c|c|c|}
\hline Planet & Molecule & $\mathrm{D} / \mathrm{H}\left(\times 10^{-5}\right)$ & Reference \\
\hline Jupiter & $\mathrm{H}_{2}$ & $1.0-2.9$ & Smith et al. (1989) \\
\hline Jupiter & $\mathrm{H}_{2}$ & $2.6 \pm 0.7$ & Mahaffy et al. (1998) \\
\hline Jupiter & $\mathrm{H}_{2}$ & $2.4 \pm 0.4$ & This work (from HD) \\
\hline Jupiter & $\mathrm{CH}_{4}$ & $2.4 \pm 0.7^{a}$ & Bjoraker et al. (1986) \\
\hline Jupiter & $\mathrm{CH}_{4}$ & $5.4_{-1.2}^{+2.6 a}$ & Carlson et al. (1993) \\
\hline Jupiter & $\mathrm{CH}_{4}$ & $2.2 \pm 0.7$ & This work (from $\mathrm{CH}_{3} \mathrm{D}$ ) \\
\hline Jupiter & $\mathrm{H}_{2}$ & $1.75 \pm 0.75$ & This work (from $\left.\mathrm{CH}_{3} \mathrm{D}\right)^{b}$ \\
\hline Saturn & $\mathrm{H}_{2}$ & $<5$ & Smith et al. (1989) \\
\hline Saturn & $\mathrm{H}_{2}$ & $1.85_{-0.6}^{+0.85}$ & This work (from HD) \\
\hline Saturn & $\mathrm{CH}_{4}$ & $1.7_{-0.8}^{+1.7^{\circ}}$ & Owen et al. (1986) \\
\hline Saturn & $\mathrm{CH}_{4}$ & $2.1 \pm 1.3^{c}$ & Noll \& Larson (1991) \\
\hline Saturn & $\mathrm{CH}_{4}$ & $2.0_{-0.7}^{+1.4}$ & This work (from $\mathrm{CH}_{3} \mathrm{D}$ ) \\
\hline Saturn & $\mathrm{H}_{2}$ & $2.3_{-0.8}^{+1.2}$ & Griffin et al. (1996) \\
\hline Saturn & $\mathrm{H}_{2}$ & $1.50_{-0.65}^{+1.45}$ & This work $\left(\text { from } \mathrm{CH}_{3} \mathrm{D}\right)^{b}$ \\
\hline
\end{tabular}

$\mathrm{D} / \mathrm{H}$ ratio. This value has to be compared with inferences of $(\mathrm{D} / \mathrm{H})_{\mathrm{ps}}$ from ${ }^{3} \mathrm{He} /{ }^{4} \mathrm{He}$ measurements in the solar wind. After appropriate corrections for processes in the current solar atmosphere and evolution in the solar interior, the solar wind measurement yields the protosolar $\left(\mathrm{D}+{ }^{3} \mathrm{He}\right) / \mathrm{H}$ ratio. Subtracting the protosolar ${ }^{3} \mathrm{He} / \mathrm{H}$ contribution finally yields $(\mathrm{D} / \mathrm{H})_{\mathrm{ps}}$ (e.g. Geiss 1993). Based on a critical review of several solar wind measurements, notably from Ulysses, and estimating the protosolar ${ }^{3} \mathrm{He} / \mathrm{H}$ ratio from the ${ }^{3} \mathrm{He} /{ }^{4} \mathrm{He}$ ratio in the planetary gas component of the meteorites $\left({ }^{3} \mathrm{He} /{ }^{4} \mathrm{He}=(1.5 \pm 0.3) 10^{-4}\right)$, Geiss and Gloeckler obtain $(\mathrm{D} / \mathrm{H})_{\mathrm{ps}}=(2.1 \pm 0.5) 10^{-5}$, in excellent agreement with our estimate. Adopting instead the jovian atmosphere ${ }^{3} \mathrm{He} /{ }^{4} \mathrm{He}$, as measured by Galileo $\left((1.66 \pm 0.05) 10^{-4}\right.$; Mahaffy et al. 1998), as representative of the protosolar ${ }^{3} \mathrm{He} /{ }^{4} \mathrm{He}$ would lead to a simi$\operatorname{lar}(\mathrm{D} / \mathrm{H})_{\mathrm{ps}}=(1.94 \pm 0.5) 10^{-5}$. (Using the same approach, Mahaffy et al. (1998) derived a significantly larger $(\mathrm{D} / \mathrm{H})_{\mathrm{ps}}=(2.7 \pm 0.3) 10^{-5}$ because they adopted the initial Ulysses value of ${ }^{3} \mathrm{He} /{ }^{4} \mathrm{He}$ in the solar wind from Bodmer et al. 1995). This confirms that Jupiter is a reliable indicator of the protosolar $\mathrm{D} / \mathrm{H}$.

The $(\mathrm{D} / \mathrm{H})_{\mathrm{ps}}$ we infer is only barely significantly larger than the value measured in the Local Interstellar Medium $(1.5 \pm 0.1) 10^{-5}$ (Linsky 1998; Sahu et al. 1999). As noted earlier by Mahaffy et al. (1998) and Geiss \& Gloekcler (1998), this indicates a modest decrease of deuterium in the part of the Galaxy where the Solar System was formed. With $(\mathrm{D} / \mathrm{H})_{\mathrm{ps}}=(2.1 \pm 0.4) 10^{-5}$, the consumption of deuterium over the last $4.55 \mathrm{Gyr}$ amounts to only $29_{-23}^{+25} \%$. If ones assumes a constant destruction rate, the extrapolation to 14 Gyr backwards gives a primordial $\mathrm{D} / \mathrm{H}$ ratio of $(1.8-4.8) 10^{-5}$. This appears to be fully consistent with values inferred from quasars at high redshifts (about $3.410^{-5}$, Kirkman et al. 2000). The assumption of constant destruction rate of deuterium with time may however not be realistic given that the stellar formation rate appears to show a peak near $z=1.5$ (Madau et al. 1998).

Within error bars, our inferred $\mathrm{D} / \mathrm{H}$ ratios for Jupiter and Saturn are indistinguishable, as we infer a Saturn/Jupiter D/H ratio of $0.75_{-0.27}^{+0.53}$. According to the interior models of Guillot (1999), this ratio is expected to be $\sim 1.15-1.35$, because Saturn's deuterium abundance was more enhanced than Jupiter's by the mixing of solar nebula gas with D-rich ices. Within error bars, the two results are consistent, but the agreement is not optimum. We also note that inspection of Table 2 suggests that most individual measurements, including ours, give higher central values for $\mathrm{D} / \mathrm{H}$ in Jupiter than in Saturn (both in $\mathrm{H}_{2}$ and in $\mathrm{CH}_{4}$, by about (0.2-0.5) $10^{-5}$ ), although the uncertainty domains always overlap.

We recognize that it seems difficult to imagine a mechanism that would deplete Saturn's atmosphere in deuterium relative to Jupiter. Saturn's atmosphere appears depleted in helium, and the most recent $\mathrm{He} / \mathrm{H}$ ratios in Jupiter and Saturn (von Zahn et al. 1998; Conrath \& Gautier 2000) can be quantitatively explained by the helium unmixing in Saturn (Hubbard et al. 1999). A possibility (D. Gautier, priv. comm.), which to our knowledge has not been fully explored, would be that HD is similarly affected by such a differentiation. In any event, we stress the importance of further improving the accuracy of $\mathrm{D} / \mathrm{H}$ measurements. High signal-to-noise observations of the Giant Planets in the HD $R(0)$ and $R(1)$ lines from the PACS instrument aboard Herschel, to be launched in 2007, may bring future insight in this problem.

Acknowledgements. This study is based on observations with ISO, an ESA project with instruments funded by ESA Member States (especially the principal investigators countries: France, Germany, The Netherlands and the UK), and with participation of ISAS and NASA. We are indebted to Françoise 
Combes and to Daniel Gautier, "Prof. Deuterium", for useful discussions.

\section{References}

Beer, R., \& Taylor, F. W. 1973a, ApJ, 179, 309

Beer, R., \& Taylor, F. W. 1973b, ApJ, 182, L131

Beer, R., \& Taylor, F. W. 1978, ApJ, 219, 763

Bézard, B., \& Gautier, D. 1985, Icarus, 61, 296

Bézard, B., Gautier, D., \& Marten, A. 1986, A\&A, 161, 387

Bjoraker, G. L., Bézard, B., Gautier, D., \& Courtin, R. 1985, BAAS, 3, 696

Bjoraker, G. L., Larson, H. P., \& Kunde, V. G. 1986, Icarus, 66,576

Bodmer, R., Bochsler, P., Geiss, J., von Steiger, R., \& Gloeckler, G. 1995, Space Sci. Rev., 72, 61

Brown, L. R., \& Paterson, D. B. 1994, J. Mol. Spectro, 168, 593

Carlson, B. E., Lacis, A. A., \& Rossow, W. B., J. Geophys. Res., 98, 5251

Clegg, P. E., et al. 1996, A\&A, 315, L38

Conrath, B. J., \& Gautier, D. 2000, Icarus, 144, 124

Conrath, B. J., \& Pirraglia, J. A. 1983, Icarus, 53, 286

Conrath, B. J., Gierasch, P. J., \& Ustinov, E. A. 1998, Icarus, 135,501

Conrath, B. J., \& Gierasch, P. J. 1986, Icarus, 67, 444

Courtin, R., Gautier, D., Marten, A., Bézard, B., \& Hanel, R. 1984, ApJ, 287, 899

Davis, G. R., Griffin, M. J., Naylor, D. A., et al. 1996, A\&A, 315, L393

de Graauw, T., et al. 1996, A\&A, 315, L49

de Graauw, T., et al. 1996, A\&A, 321, L13

Drakopoulos, P. G., \& Tabisz, G. C. 1987a, Phys. Rev. A, 36, 5556

Drakopoulos, P. G., \& Tabisz, G. C. 1987b, Phys. Rev. A, 36, 5566

Drossart, P., Encrenaz, T., Combes, M., Kunde, V., \& Hanel, R. 1982, Icarus, 49, 416

Drossart, P., Fouchet, T., Crovisier, J., et al. 1999, The Universe as sen by ISO, ESA SP-427, 169

Encrenaz, T., et al. 1996, A\&A, 315, L397

Fegley, B., \& Prinn, R. G. 1988, ApJ, 324, 621

Feuchtgruber, H., Lellouch, E., Bézard, B., et al. 1999, A\&A, 341, L17

Gautier, D., Bézard, B., Marten, A., et al. 1982, ApJ, 257, 901

Geiss, J. 1993, in Origin and evolution of the Elements (Cambridge Univ. Press), 89

Geiss, J., \& Gloeckler, G. 1998, Space Sci. Rev., 84, 239

Gezari, D. Y., Mumma, M. J., Espenak, F., et al. 1989, Nature, 342,777

Griffin, M. J., et al. 1996, A\&A, 315, L389

Guillot, T. 1999, Planet Space Sci., 47, 1183

Hanel, R., Conrath, B., Flasar, F. M., et al. 1981, Science, 212, 192

Hartmann, J.-M., Boulet, C., Fouchet, T., \& Drossart, P., Submitted to JQSRT
Hubbard, W. B., Porco, C. C., Hunten, D. M., et al. 1997, Icarus, 130, 404

Jennings, D. E., Weber, A., \& Brault, J. W. 1987, J. Mol. Spectro., 126, 19

Kessler, M. F., et al. A\&A, 315, L27

Kirkman, D., Tytler, D., Burles, S., Lubin, D., \& O'Meara, J. M. 2000, ApJ, 529, 655

Knacke, R. F., Kim, S. J., Ridgway, S. T., \& Tokunaga, A. T. 1982, ApJ, 262, 388

Kunde, V., et al. 1982, ApJ, 263, 443

Lecluse, C., Robert, F., Gautier, D., \& Guiraud, M. 1996, Planet Space Sci., 44, 1579

Lévy, A., Lacome, N., \& Tarrago, G. 1993, J. Mol. Spectro., 157,172

Jacquinet-Husson, N., Arié, E., Ballard, J., et al. 1999, J. Quant. Spectrosc. Rad. Trans., 62, 205

Lellouch, E., et al. 1996, BAAS, 28, 2234

Lellouch, E., et al. 1998, International Jupiter-Saturn Symposium, Nantes, 11-15 May 1998

Lindal, G. F., Sweetnam, D. N., \& Eshleman, V. R. 1985, AJ, 90, 1136

Linsky, J. L. 1998, Space Sci. Rev., 84, 285

Madau, P., Pozzetti, L., \& Dickinson, M. 1998, ApJ, 498, 106

Mahaffy, P. R., Donahue, T. M., Atreya, S. K., Owen, T. C., \& Niemann, H. B. 1998, Space Sci. Rev., 84, 251

Moses, J. I., Bézard, B., Lellouch, E., et al. 2000a, Icarus, 143, 244

Moses, J. I., Lellouch, E., Bézard, B., et al. 2000b, Icarus, 145, 166

Niemann, H., et al. 1996, Science, 272, 846

Noll, K. S., \& Larson, H. P. 1991, Icarus, 89, 168

Ollivier, J. L., Billebaud, F., Drossart, P., et al. 2000, A\&A, 356,347

Owen, T., Lutz, B. L., \& de Bergh, C. 1986, Nature, 320, 2440

Poll, J. D., \& Wolniewicz, L. 1978, J. Chem. Phys., 68, 3053

Roos-Serote, M. 1997, Ph.D. Thesis, Université Paris-7

Roos-Serote, M., Drossart, P., Encrenaz, T., et al. 1999, Icarus, 137,315

Sahu, M. S., Landsman, W., Bruhweiler, F. C., et al. 1999, ApJ, 523, L159

Salama, A., et al. 1997, First ISO workshop on analytical spectroscopy, ESA SP-419, 17

Smith, M. D., Conrath, B. J., \& Gautier, D. 1996, Icarus, 124, 598

Smith, M. D. 1998, Icarus, 132, 176

Smith, W. H., Schempp, W. V., \& Baines, K. H. 1989, ApJ, 326,967

Stevenson, D. J. 1982, Planet Space Sci., 30, 755

Tarrago, G., Dang-Nhu, M., \& Goldman, A. 1981, J. Mol. Spectro., 88, 311

Trauger, J. T., Roesler, F. L., Carleton, N. P., \& Traub, W. A. 1973, ApJ, 184, L137

Ulivi, L., et al. 1991, ApJ, 378, L29

Weisstein, E. E., \& Serabyn, E. 1994, Icarus, 109, 367

von Zahn, U., Hunten, D. M., \& Lehmacher, G. 1998, J. Geophys. Res., 103, 22815 